\title{
STING controls Herpes Simplex Virus in vivo independent of type I interferon induction
}

Lívia H. Yamashiro ${ }^{1,2}$, Stephen C. Wilson ${ }^{1,3}$, Huntly M. Morrison ${ }^{1}$, Vasiliki Karalis ${ }^{4}$, Jing-Yi J. Chung ${ }^{1}$, Katherine J. Chen ${ }^{1}$, Helen S. Bateup ${ }^{4,5,6}$, Moriah L. Szpara ${ }^{7}$, Angus Y. Lee $^{8}$, Jeffery S. Cox ${ }^{1,9}$, Russell E. Vance ${ }^{1,2,8,9^{*}}$

${ }^{1}$ Division of Immunology and Pathogenesis, Department of Molecular and Cell Biology, University of California, Berkeley, CA 94720 USA

${ }^{2}$ Howard Hughes Medical Institute, University of California, Berkeley, CA 94720 USA

${ }^{3}$ Current Address: Celgene Corporation, 200 Cambridge park Dr, Cambridge, MA 02140

${ }^{4}$ Division of Neurobiology, Department of Molecular and Cell Biology, University of California, Berkeley, CA 94720 USA

${ }^{5}$ Helen Wills Neuroscience Institute, University of California, Berkeley, CA 94720 USA

${ }^{6}$ Chan Zuckerberg Biohub, San Francisco, CA 94158 USA

${ }^{7}$ Department of Biochemistry and Molecular Biology, Center for Infectious Disease

Dynamics, Huck Institutes of the Life Sciences, Pennsylvania State University, University Park, PA 16801 USA

${ }^{8}$ Cancer Research Laboratory, University of California, Berkeley, CA 94720 USA

${ }^{9}$ Henry Wheeler Center for Emerging and Neglected Diseases, University of California, Berkeley, CA 94720 USA

* e-mail: rvance@berkeley.edu 


\begin{abstract}
The Stimulator of Interferon Genes (STING) pathway initiates potent immune responses upon recognition of DNA derived from bacteria, viruses and tumors. To signal, the Cterminal tail (CTT) of STING recruits TBK1, a kinase that phosphorylates serine 365 (S365) in the CTT. Phospho-S365 acts as a docking site for IRF3, a transcription factor that is phosphorylated and activated by TBK1, leading to transcriptional induction of type I interferons (IFNs). IFNs are essential for antiviral immunity and are widely viewed as the primary output of STING signaling in mammals. However, other more evolutionarily ancestral responses, such as induction of NF- $\mathrm{B}$ or autophagy, also occur downstream of STING. The relative importance of the various outputs of STING signaling during in vivo infections is unclear. Here we report that mice harboring a serine 365-to-alanine (S365A) point mutation in STING exhibit normal susceptibility to Mycobacterium tuberculosis infection but, unexpectedly, are resistant to Herpes Simplex Virus (HSV)-1, despite lacking STING-induced type I IFN responses. Likewise, we find $\operatorname{Irf} 3^{-/-}$mice exhibit resistance to HSV-1. By contrast, resistance to HSV-1 is abolished in mice lacking the STING CTT or TBK1, suggesting that STING protects against HSV-1 upon TBK1 recruitment by the STING CTT, independent of IRF3 or type I IFNs. Interestingly, we find that STING-induced autophagy is a TBK1-dependent IRF3-independent process that is conserved in the STING S365A mice, and autophagy has previously been shown to be required for resistance to $\mathrm{HSV}-1$. We thus propose that autophagy and perhaps other ancestral interferon-independent functions of STING are required for STING-dependent antiviral responses in vivo.
\end{abstract}




\section{Introduction}

The immune response to pathogens is initiated upon detection of pathogenassociated molecular patterns (PAMPs) such as lipopolysaccharide, flagellin and nucleic acids [1]. Double-stranded DNA (dsDNA) is an important PAMP for the detection of many pathogens, including Mycobacterium tuberculosis and Herpes Simplex Virus-1 (HSV-1) [2-4]. In vertebrates, the intracellular presence of dsDNA is detected by cyclicGMP-AMP Synthase (cGAS), a dsDNA-activated enzyme that produces a cyclic

8 dinucleotide (CDN) second messenger called 2'3'-cyclic-GMP-AMP (2'3'cGAMP) [5-

9 10]. 2'3'cGAMP binds and activates the ER-resident transmembrane protein Stimulator

10 of Interferon Genes (STING). Transcriptional induction of type I IFNs is widely

11 presumed to be the primary output of STING signaling during antiviral defense.

12 However, STING is evolutionarily ancient, present even in bacteria [11] and in animals

13 such as the starlet sea anemone Nematostella vectensis and Drosophila melanogaster that

14 do not appear to encode type I interferons [12]. By contrast, autophagy and NF- $\kappa \mathrm{B}$

15 signaling are ancestral STING-induced signaling pathways, present in both $N$. vectensis

16 and D. melanogaster, raising the possibility that these pathways are the primary or

17 ancestral signaling outputs of STING [13-16].

The relative in vivo importance of the various signaling outputs of STING for

19 anti-viral immunity in vertebrates is unknown. To address this issue, we used

20 CRISPR/Cas9 to generate two distinct Sting mutant mouse lines: (1) STING S365A

21 mice, which harbor a mutation in Sting that results in a serine to alanine substitution at

22 amino acid 365; and (2) STING $\Delta$ CTT mice, in which valine 340 has been substituted by

23 a STOP codon, resulting in a STING protein that lacks the entire CTT (Supp. Fig. S1a 
and S1b). We compared the S365A and $\Delta \mathrm{CTT}$ mice to our previously generated STINGnull Goldenticket (Gt) mice [17]. Since phosphorylation of S365 in the CTT of STING is required for the recruitment and activation of IRF3 [18-20], we predicted that S365A mice would be deficient in type I IFN responses downstream of STING, but would retain all other STING-dependent signaling events such as autophagy or NF-kB induction. The STING CTT contains S365 and is also essential for recruitment of TBK1 [21, 22]. Thus, we predicted that $\triangle \mathrm{CTT}$ mice should also be deficient in all TBK1-dependent responses downstream of STING.

In the present study, we found that STING mutations do not affect susceptibility to M.tuberculosis, while control of HSV-1 infection requires the STING CTT but, unexpectedly, is largely independent of S365- or IRF3-induced type I IFNs. Control of HSV-1 also required TBK1, suggesting that STING protects against HSV-1 upon TBK1 recruitment by the STING CTT, independent of IRF3 or type I IFNs. We found that STING-induced autophagy is a TBK1-dependent IRF3-independent process that is conserved in the STING S365A mice. Thus, our data provide in vivo support for the idea that autophagy induction and perhaps other ancestral interferon-independent functions of STING may be preserved in vertebrates for host defense.

\section{Results}

\section{Defective type I IFN induction in STING S365A and $\Delta$ CTT macrophages.}

Prior studies identified serine 365 of mouse STING (S366 in human STING) to be essential for STING-induced type I IFN expression in transfected or transduced cells in vitro [18-20]. To test whether endogenous STING requires the CTT and S365 for IFN 
47 induction in primary cells, bone marrow-derived macrophages from wild-type (WT)

48 C57BL/6J, Goldenticket (Gt) STING null mice, and STING S365A and $\Delta$ CTT mice were

49 stimulated with STING-specific agonists, including CDNs such as c-di-GMP and

50 2'3'cGAMP, as well as the cGAS agonist, dsDNA. As controls, cells were also stimulated

51 with Sendai virus (SeV) and poly I:C, which induce type I IFNs via the RIG-I-MAVS

52 pathway, independently of cGAS-STING. As expected, stimulation with STING-specific

53 agonists resulted in increased Ifnb expression only in WT cells and not in any of the

54 STING mutant cells. By contrast, the IFN response of all four genotypes was similar in

55 response to $\mathrm{SeV}$ and poly I:C (Fig. 1a). STING activation can also lead to production of

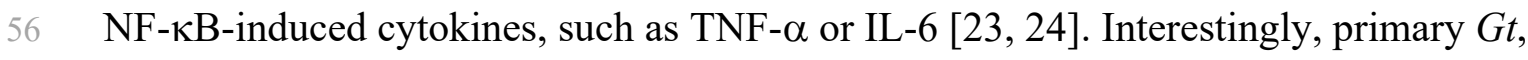

57 S365A and $\triangle \mathrm{CTT}$ macrophages stimulated in vitro with CDNs or dsDNA were defective

58 for TNF- $\alpha$ induction as compared to WT cells (Supp. Fig. 1c). However, in vivo

59 stimulation with 5,6-dimethylxanthenone-4-acetic acid (DMXAA), a potent STING

60 agonist $[25,26]$, resulted in measurable TNF- $\alpha$ responses in the serum of WT and

61 STING S365A mice, whereas $G t$ and $\Delta$ CTT mice were defective in TNF- $\alpha$ production as

62 expected (Fig. 1b). As a control, the TNF- $\alpha$ response to STING-independent stimuli (e.g.,

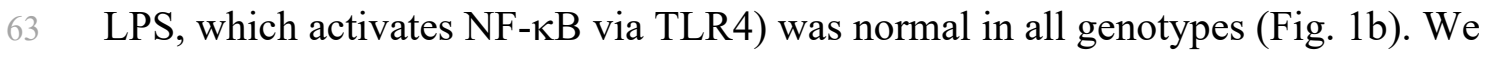

64 conclude that $\mathrm{S} 365$ may play a role in NF- $\mathrm{B}$ activation, at least in macrophages, but is

65 not required for NF- $\mathrm{KB}$ activation in vivo in response to strong STING agonists.

68 immunoblotting (Fig. 1c). The STING S365A mutation did not affect expression of the

69 STING protein itself or downstream components such as TBK1 and IRF3. STING $\Delta$ CTT 
70 mice harbor a STING protein of the expected (decreased) molecular weight.

71 Phosphorylation of TBK1 — but not of STING or IRF3 - occurred in S365A cells in

72 response to STING agonist, consistent with the generally accepted requirement for S365

73 phosphorylation for IRF3 binding and activation (Fig. 1c). By contrast, no

74 phosphorylation of STING, TBK1 or IRF3 was seen in $\triangle \mathrm{CTT}$ cells, as expected.

In addition to its role in IFN-induction, TBK1 has previously been shown to

76 activate autophagy via the phosphorylation of autophagy adaptor proteins such as

77 NDP52, p62 and optineurin [27]. Likewise, STING activation itself is associated with

78 autophagy-like responses [16, 19, 28, 29]. Interestingly, a recent report claimed that

79 STING-induced autophagy does not require the CTT or TBK1 [16]; however, these

80 experiments utilized conditions that may not reflect the true in vivo requirements, such as

81 overexpressed proteins, immortalized cell lines, and/or artificial in vitro stimulations. In

82 order to investigate whether S365 or the CTT is required for endogenous STING to

83 activate autophagy-like processes, primary macrophages were transfected with

84 2'3'cGAMP and conversion of LC3-B from form I to the lipidated form II was analyzed.

85 Robust LC3-B conversion was observed in WT and S365A cells, while this response was

86 reduced in $G t$ and $\Delta$ CTT cells (Fig. 1d and e, Supp. Fig. S1d), indicating that STING-

87 dependent autophagy is independent of S365A-IRF3 activation and type I IFN responses

88 but largely requires the CTT. To confirm this result, we quantified colocalization of LC3

89 puncta and cytosolic DNA. Primary macrophages were transfected for $6 \mathrm{~h}$ with Cy3-

90 labeled DNA and colocalization with LC3 puncta was quantified by

91 immunofluorescence. STING-deficient $G t$ and $\Delta$ CTT cells exhibited poor colocalization

92 of DNA and LC3, whereas WT and S365A cells exhibited robust and indistinguishable 
93 DNA-LC3 colocalization (Fig. If and Supp. Fig. S1e). Taken together these data indicate

94 that endogenous STING requires S365 for IRF3 recruitment and induction of type I IFNs

95 downstream of STING, whereas the CTT (but not S365) is required for TBK1

96 recruitment and robust autophagy induction. Our new mouse models therefore allow us to

97 genetically separate the IFN- and autophagy-inducing functions of endogenous STING

98 for the first time. 


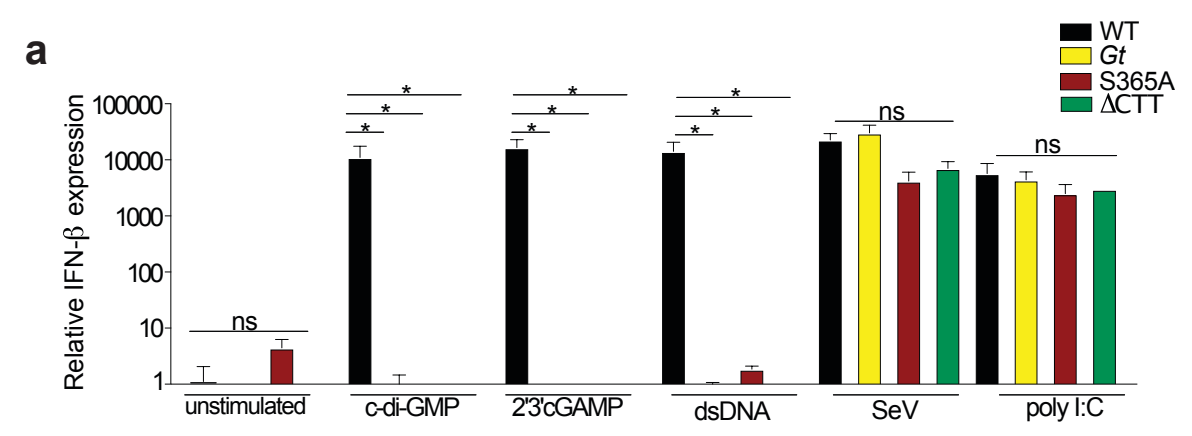

b

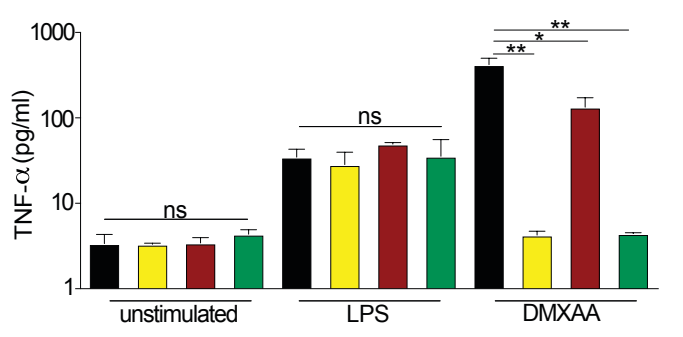

C
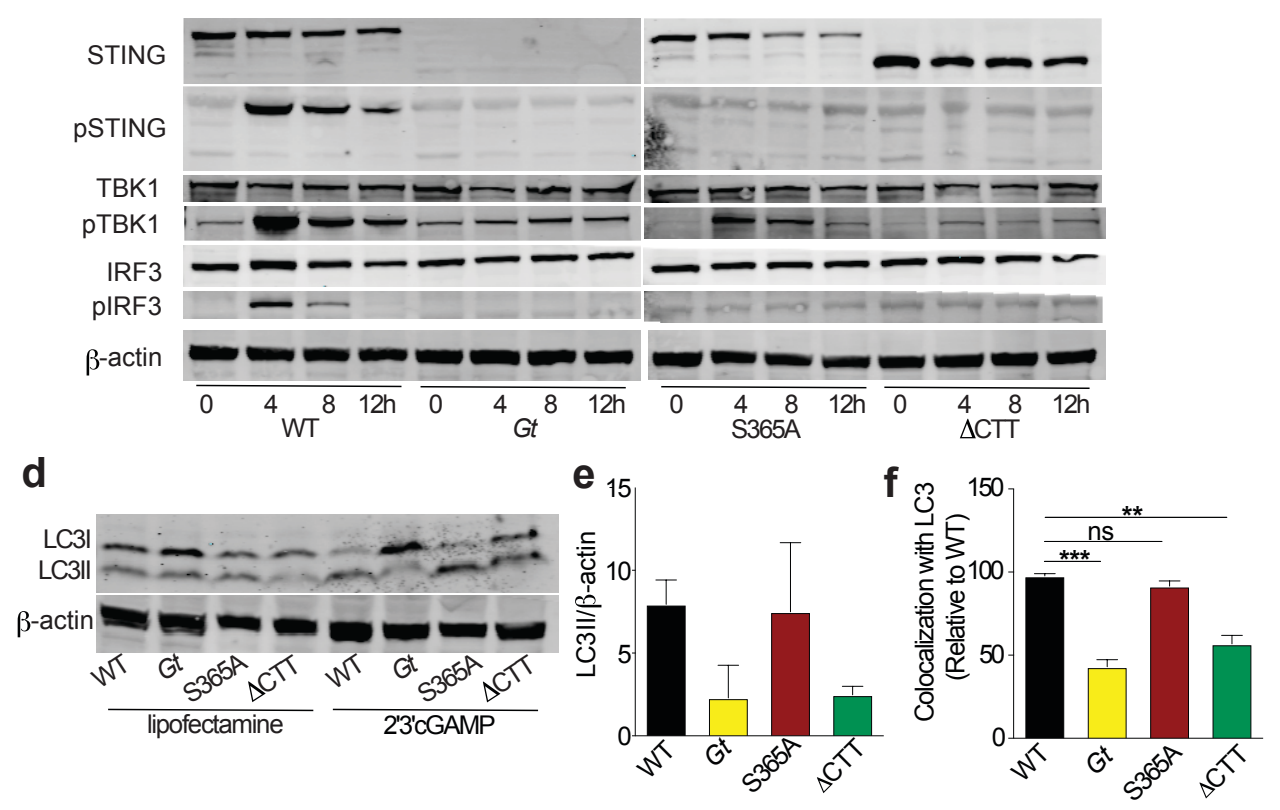

104

105

106

Figure 1. Defective type I IFN induction in STING S365A and $\triangle$ CTT macrophages. a, Bone marrow derived macrophages were stimulated for $6 \mathrm{~h}$ and relative expression of IFN- $\beta$ mRNA was measured. b, Mice were injected DMXAA $(25 \mathrm{mg} / \mathrm{kg}$, i.p.) or LPS (10 ng, i.v.) and TNF- $\alpha$ production was measured on the serum $2 \mathrm{~h}$ later. c, Primary macrophages were transfected with dsDNA for 4,8 or $12 \mathrm{~h}$ or $\mathbf{d}, 2^{\prime} 3^{\prime} \mathrm{cGAMP}$ for $6 \mathrm{~h}$, and cell lysates were analyzed by immunoblotting for the indicated proteins. e, Quantification of (d). f, Quantification of LC3-DNA colocalization in primary macrophages transfected with Cy3-DNA for 6h and stained with LC3. Images were analyzed by an automated pipeline created on Perkin Elmer Harmony software for colocalization quantification (for more details refer to Methods). Representative results of three independent experiments. Error bars are SEM. Analyzed with one-way ANOVA and Tukey post-test. *, $\mathrm{p} \leq 0.05$; $* *, \mathrm{p} \leq 0.005 ; * * *, \mathrm{p} \leq 0.0001$. ns, not significant. 


\section{STING mutant mice exhibit normal susceptibility to M. tuberculosis infection.}

To determine whether STING-induced interferons and autophagy have distinct

functions during in vivo infection, we first examined infections with the bacterium $M$.

tuberculosis. Previous reports have suggested that the cGAS-STING pathway detects $M$. tuberculosis in macrophages and initiates both a type I IFN response and autophagy-like colocalization of bacteria with LC3 [4, 30-32]. Type I IFNs exacerbate many bacterial infections, including M. tuberculosis infection [33-36] whereas autophagy is generally anti-bacterial [37]. Therefore, loss of STING may have counteracting effects that obscure its function; indeed, STING-null Gt mice do not exhibit dramatic alterations in susceptibility to M. tuberculosis infection $[31,38]$. We hypothesized that perhaps STING S365A mice, which are defective for STING-induced type I IFN induction but not for autophagy, might exhibit enhanced resistance to M. tuberculosis. Consistent with this hypothesis, $\operatorname{Irf}^{-/-}$mice have previously been reported to be resistant to M.tuberculosis [30]. Therefore, we aerosol infected mice harboring WT, Gt, S365A, or $\Delta \mathrm{CTT}$ STING alleles with virulent M.tuberculosis. We found that all STING genotypes were similarly susceptible to M.tuberculosis with similar survival rates, bacterial burdens in lungs and spleens, and cytokine production (Fig. 2a-1). 
a

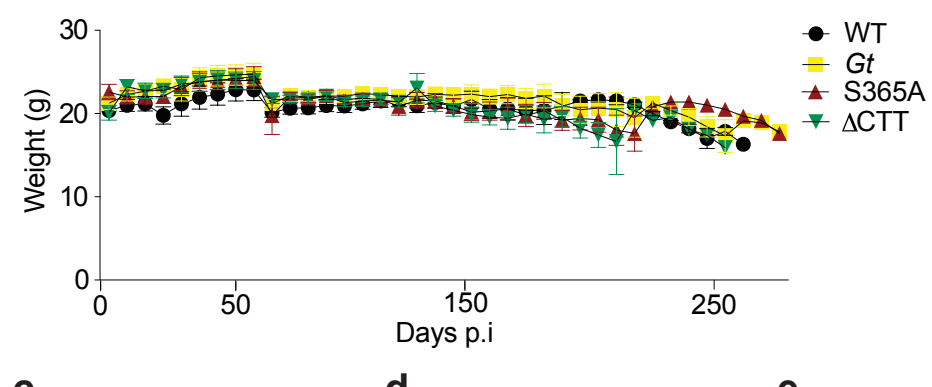

b

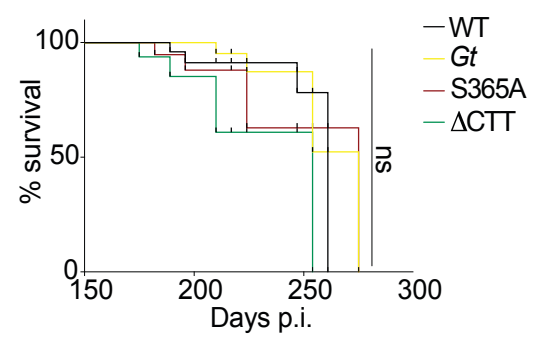

f
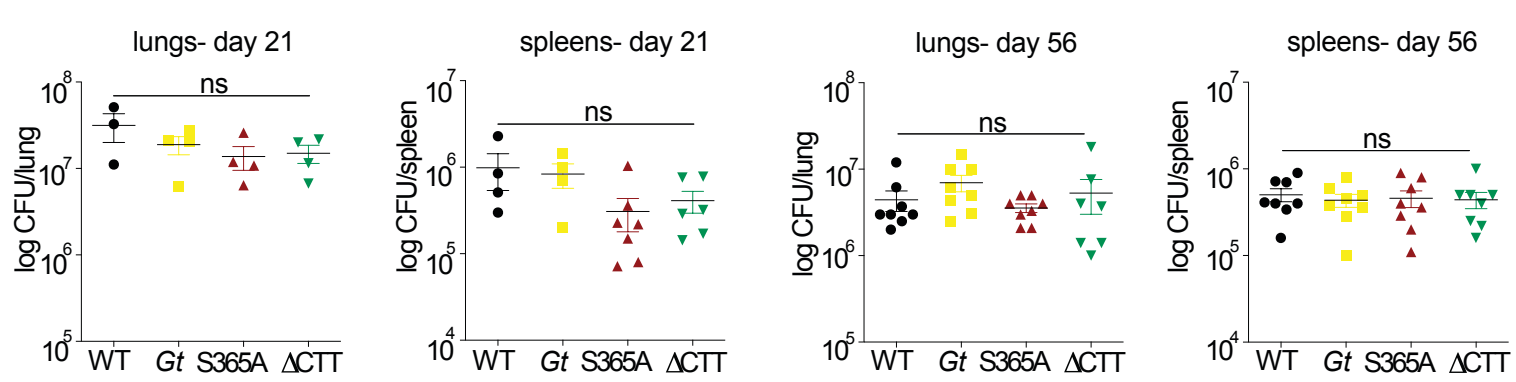

g

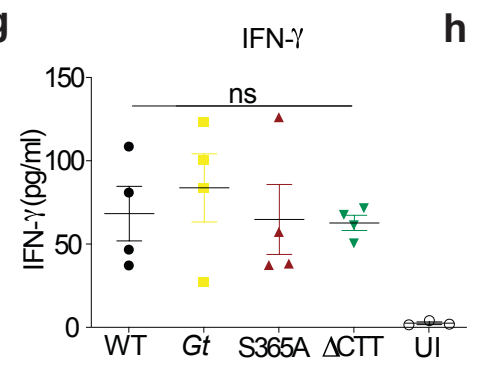

j

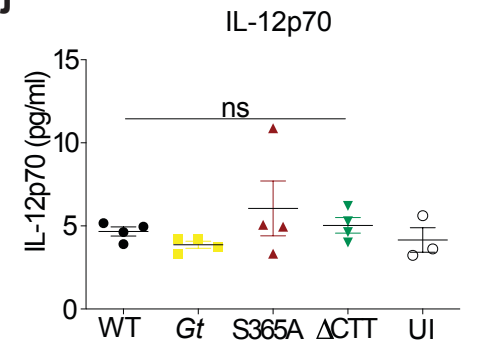

h
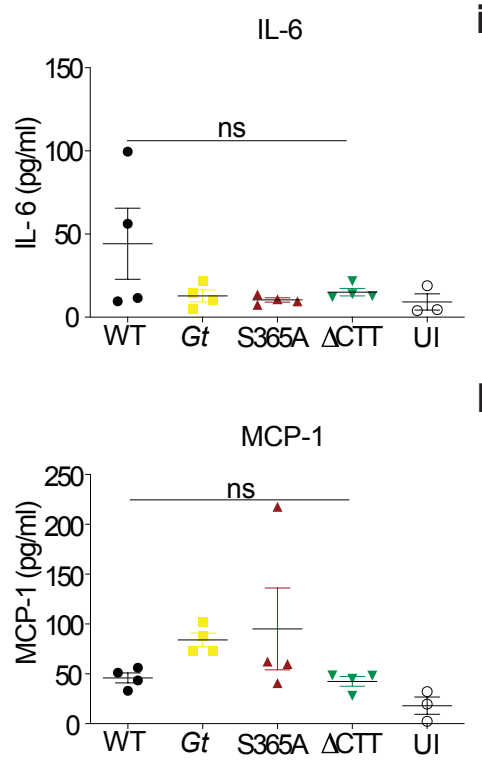

i
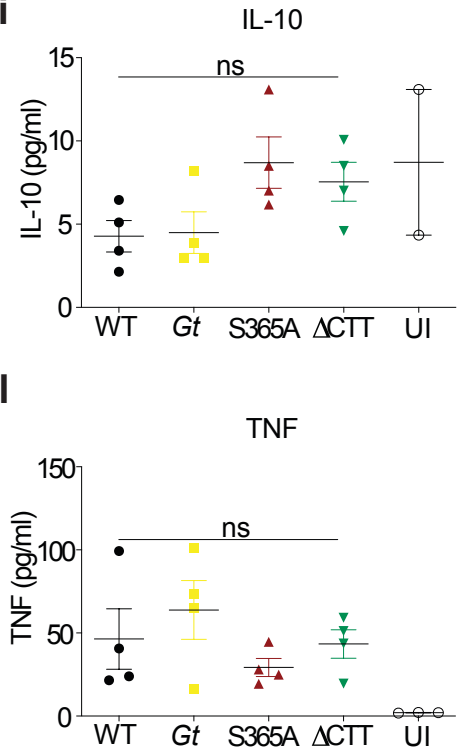

Fig 2. STING mutant mice exhibit normal susceptibility to M.tuberculosis infection. Mice were aerosol infected with $400 \mathrm{CFU}$ dose of M. tuberculosis (Erdman strain) and a, weighed every week. b, Survival of infected mice. c, Bacterial burden from lungs and d, spleens at 21 and e-f, 56 days post infection. $\mathbf{g}-\mathbf{l}$, Cytokine levels in the lungs from infected mice at day 21 , measured by CBA. Similar (not statistically different among genotypes) results were observed at day 56 (data not shown). All mice except C57BL/6J WT were bred in-house. Representative results of five independent experiments. Error bars are SEM. Analyzed with one-way ANOVA and Tukey post-test. ns, not significant. 
To confirm that STING-induced type I IFN signaling does not affect $M$. tuberculosis susceptibility, we also sought to infect mice lacking the downstream

150 transcription factor, IRF3. However, the published $\mathrm{Irf}^{-/-}$mice that were previously

151 tested are also deficient in $B c l 2 l 12$, a gene that neighbors $\operatorname{Irf} 3$ and that was inadvertently

152 disrupted by the deletion targeting $\operatorname{Irf3}$ [39]. Therefore, we generated new $\operatorname{Irf3}$ deficient

153 (but $B c l 2 l 12^{+/+}$) mice, as well as Bcl2l12 $2^{-/-}$(but $\operatorname{Irf} 3^{+/+}$) mice, using CRISPR-Cas9

154 (Supp. Fig. S2a-S2d). We found $\operatorname{Irf} 3^{-/-}$mice, Bcl2ll2 $2^{-/-}$mice, and the previously tested

155 doubly deficient mice, were all similarly susceptible to M.tuberculosis as WT mice

156 (Supp. Fig. S2e-f). We cannot explain the previously reported resistance of $\operatorname{Irf} 3^{-/}$mice

157 but suspect this may be related to microbiota differences between $\operatorname{Irf3}^{-/-}$lines.

158 Nevertheless, we conclude that although $M$. tuberculosis can activate cGAS-STING-

159 IRF3 in macrophages in vitro, STING does not appear to play significant beneficial or

160 detrimental roles in M.tuberculosis pathogenesis in vivo.

\section{S365A mice are resistant to systemic HSV-1 infection.}

Given that STING is essential for resistance to HSV-1, we next decided to

164 challenge our STING mutant mice with HSV-1. Sting-deficient mice are highly

165 susceptible to HSV-1 infection [40-42]. Although induction of type I IFN is presumed to

166 be a major mechanism of STING-mediated protection against HSV-1, the relative

167 importance of type I IFNs and other STING-dependent responses in host defense against

168 HSV-1 has not been resolved. Indeed, the immune response to HSV-1 is complex and

169 multi-factorial. HSV-1 encodes factors to block the type I IFN response, perhaps limiting

170 its effectiveness in control of the infection [41, 43, 44]. Moreover, it has been shown that 
neurons do not require type I IFNs — and can instead rely on autophagy — to limit HSV-1 replication in mice in vivo and in vitro [45]. These observations led us to hypothesize that interferon-independent signaling downstream of STING may contribute to control of HSV-1.

Initially, mice were intravenously infected with HSV-1 (KOS strain). As expected, WT mice were resistant to infection and remained healthy through 12 days post infection, whereas STING-deficient $G t$ mice were very susceptible to infection and exhibited rapid weight loss and complete paralysis, succumbing 6 days post infection (Fig. 3a-c) [41]. The $\Delta$ CTT mice phenocopied the susceptibility of Gt mice, demonstrating that the STING CTT is critical for defense against HSV-1. However, in contrast to $\triangle \mathrm{CTT}$ mice, the S365A mice unexpectedly showed marked resistance to infection, exhibiting only limited weight loss and paralysis, and recovering fully after 6 days of infection (Fig. 3a-c). Susceptibility of $G t$ and $\Delta$ CTT mice correlated with elevated viral titers in the brains and spinal cords compared to reduced titers in resistant WT and S365A tissues (Fig. 3d and e). Viral titers among all four genotypes were

186 similarly low in the liver, confirming the neurotropism of HSV-1 (Supp. Fig. S3a). Given 187 that type I IFNs are essential for resistance to HSV-1 [46, 47], and that STING is required 188 for type I IFN induction to HSV-1 [40-42, 48], we were surprised that S365A mice were 189 not as susceptible to infection as $G t$ and $\Delta$ CTT mice. One possibility to explain this result 190 is that S365A is not required for STING-dependent type I IFN induction in vivo. To test 191 this possibility, we measured expression of Ifnb and the interferon stimulated genes 192 (ISGs) viperin and Ifit1 in mice brains following intravenous infection. Only WT brains 193 exhibited a detectable STING-induced IFN response (Fig. 3f and g and Supp. Fig. S3b). 
In addition, Tnf and Il6 expression was also elevated only in the brains of WT mice (Supp. Fig. S3c and S3d). These data indicate that S365 is critical for STING-induced type I IFN and other cytokines, but surprisingly, this S365-induced response is not critical for STING-dependent immunity to HSV-1.
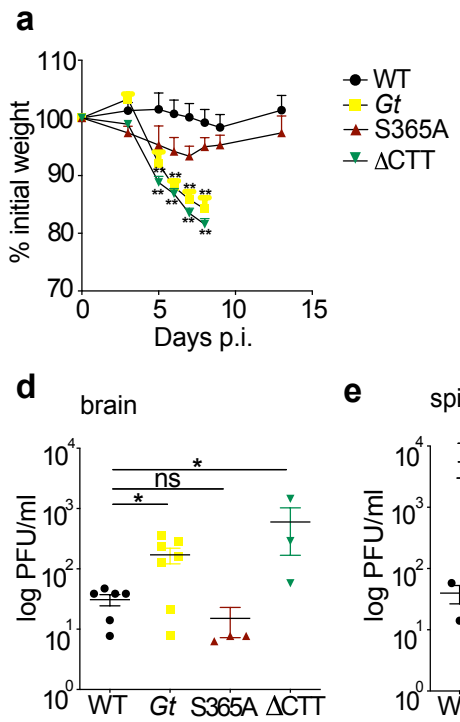

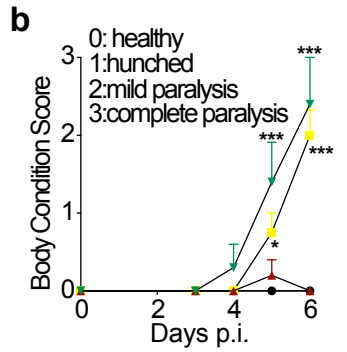

e spinal cord

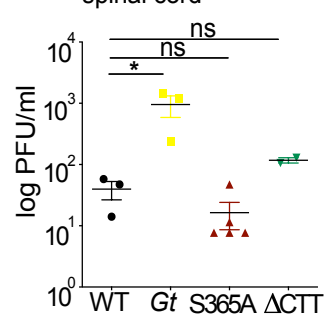

f

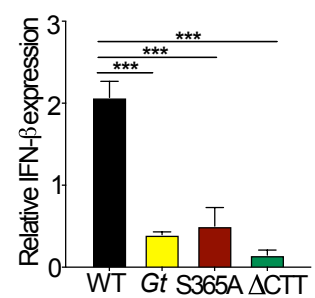

c

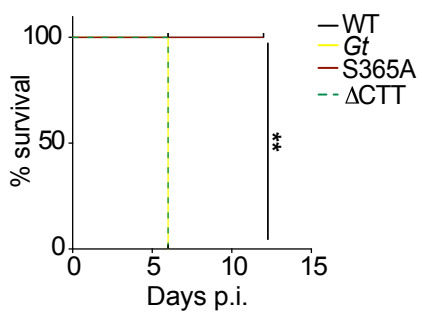

g

Figure 3. S365A mice are resistant to systemic HSV-1 infection. Mice were intravenously infected with $1 \times 10^{6}$ PFU of HSV-1 (KOS strain). a, Percentage of initial weight following infection. b, Body condition score (BCS) of infected mice. c, Survival of mice following infection. d, Viral titers in the brain and e, spinal cord at 6 days p.i. f, Relative expression of Ifnb and $\mathbf{g}$, Viperin in the brain at 3 days p.i. All mice except C57BL/6J WT were bred in-house. Representative of at least three independent experiments. Error bars are SEM. Analyzed with one-way ANOVA and Tukey post-test. $*, \mathrm{p} \leq 0.05 ; * *, \mathrm{p} \leq 0.005 ; * * *, \mathrm{p} \leq 0.0001$. ns, not significant.

\section{S365A mice are resistant to ocular HSV-1 infection.}

$\mathrm{HSV}-1$ is a neurotropic virus that is transmitted via mucosal routes (typically oral, ocular, or genital) and infects epithelial cells before reaching the central nervous system where it establishes latency in neurons $[49,50]$. Therefore, in order to mimic a more natural route of infection, we challenged mice with HSV-1 using an eye infection model $[41,51]$. In these experiments, we used strain 17, a more virulent HSV-1 isolate, because 
214 the KOS strain used for intravenous infections fails to cause pathology in the eye

215 infection model [51]. As with systemic infection, $G t$ and $\Delta \mathrm{CTT}$ mice rapidly lost weight

216 and all mice succumbed to infection by 6-7 days post infection (Fig. 4a, b). In contrast,

217 WT mice remained fully resistant and S365A mice presented an intermediate phenotype,

218 with initial weight loss but later recovery and $\sim 50 \%$ survival (Fig. 4a, b). Similar to

219 systemic infection, the susceptibility of the mice correlated with viral burdens: WT and

220 S365A exhibited lower viral titers in the eye wash (Supp. Fig. S4a), whole brain, brain

221 stem and spinal cord as compared to $G t$ and $\Delta$ CTT mice (Fig. 4c-e). Once again, we

222 found that Ifnb and viperin expression was elevated in WT but not in $G t$, S365A or $\Delta$ CTT

223 brain stems (Fig. 4f and Supp. Fig. S4b). Previous studies have shown that STING-

224 dependent control of HSV-1 is cell-type specific [41]. To investigate an S365-dependent

225 viral control in brain cells, we infected primary neurons and astrocytes in vitro with

226 HSV-1. However, we observed similar viral yields and autophagy-related processes

227 (colocalization of virus-LC3 and LC3 conversion) (Supp. Fig.S5a-e) in both cell

228 populations among all genotypes, confirming prior reports that STING does not function

229 cell autonomously in these cell types [42]. To address which cells require S365 for type I

230 IFN induction in vivo, we sorted brain cells (neurons, astrocytes and microglia) 3 days

231 post infection from brains of HSV-1-infected mice (ocular route). We found elevated Ifnb

232 expression in all cell populations only in WT mice (Fig. 4g-i), confirming that IFN- $\beta$

233 induction in vivo requires STING S365. Together, our data suggest that STING-mediated

234 control of HSV-1 infection in vivo does not require STING S365-induced type I IFN

235 production. 
a

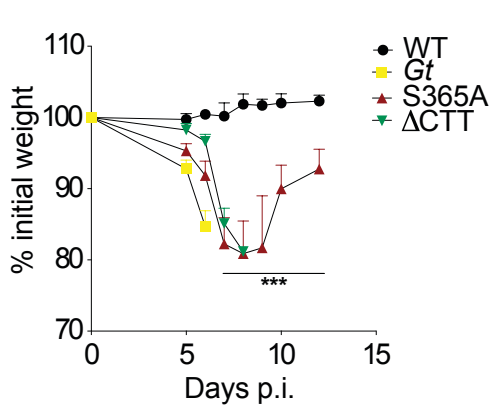

d

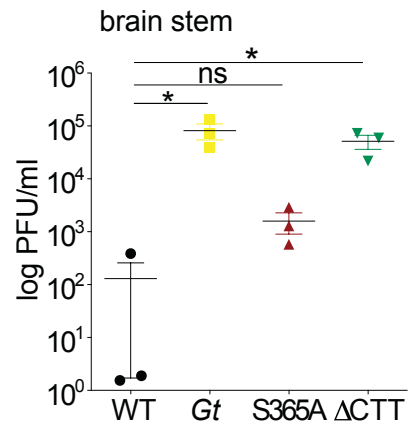

g

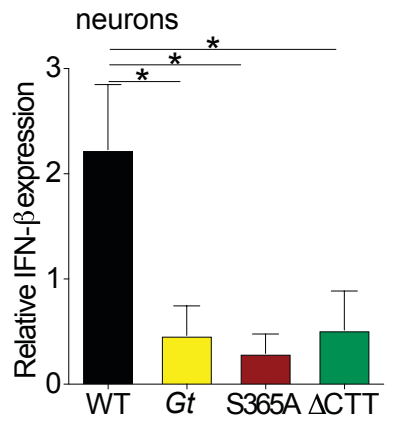

b

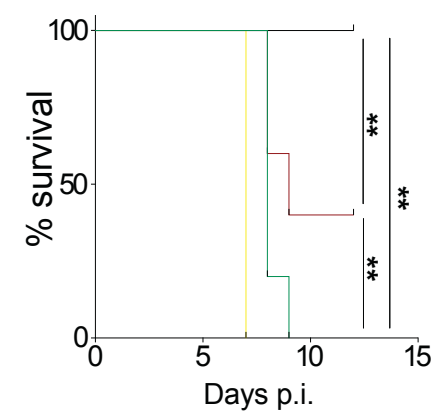

e

spinal cord

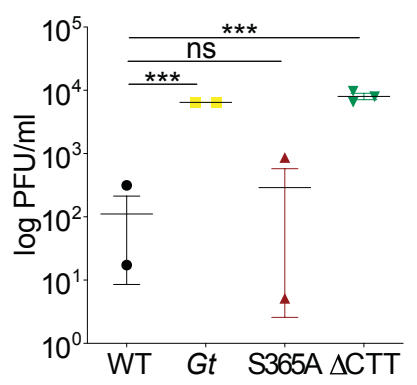

h

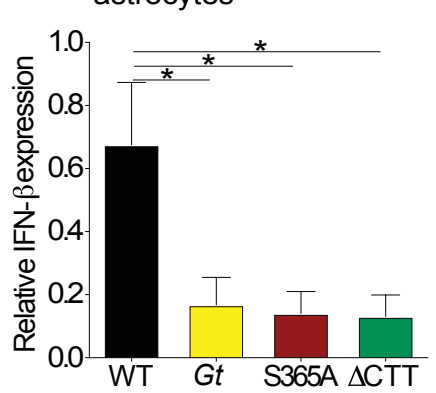

C

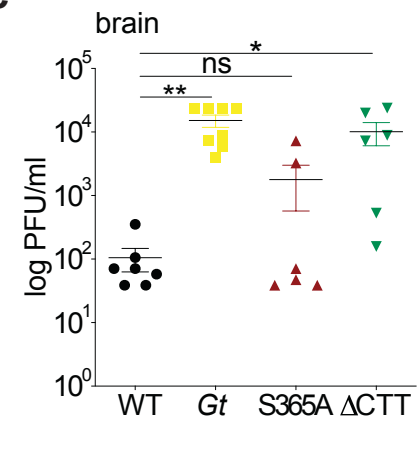

f

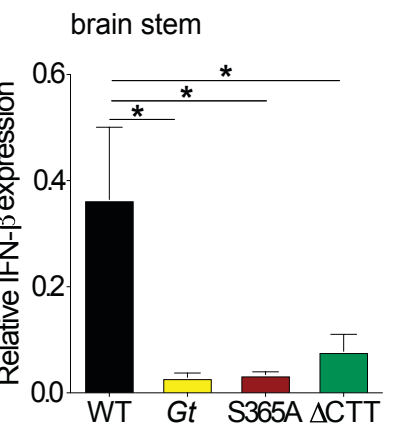

i

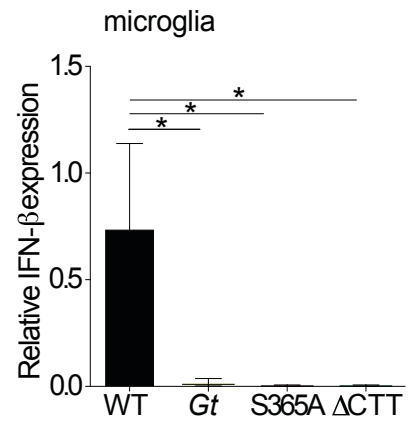

Figure 4. S365A mice are resistant to ocular HSV-1 infection. Mice were infected via the ocular route with $1 \times 10^{5} \mathrm{PFU}$ of HSV-1 (strain 17). a, Percentage of initial weight following infection. b, Survival of infected mice. c, Viral titers in the brain, $\mathbf{d}$, brain stem and e, spinal cord from 6 days p.i. f, Relative expression of If $n b$ in the brain stem at 3 days p.i. g, Brains from infected mice were collected 3 days p.i. and neurons, $\mathbf{h}$, astrocytes and $\mathbf{i}$, microglia cells were sorted, and Ifnb expression was analyzed. Representative of more than five independent experiments. Error bars are SEM. Analyzed with one-way ANOVA and Tukey post-test. *, p $\leq 0.05 ; * *, p \leq 0.005 ; * * *, p$ $\leq 0.0001$. ns, not significant.

\section{STING S365A and $\operatorname{Irf3}^{-/-}$mice phenocopy resistance to HSV-1.}

Because TBK1 has been implicated in autophagy induction $[4,52,53]$ whereas

249 IRF3 acts as a transcriptional factor to induce type I IFNs downstream of STING, we 
250 investigated the role of these proteins in the context of an in vivo infection with HSV-1.

$251 T b k 1^{-/-}$mice die as embryos, but this lethality is reversed on a $\operatorname{Tnfr} \mathrm{I}^{-/-}$background. We

252 therefore analyzed $\operatorname{Tnfr} 1^{-/-}$mice compared to $\operatorname{Tbk1^{-/}} \operatorname{Tn} f r 1^{-/-}$double deficient mice.

$253 \operatorname{Tbk1}^{-/-} \operatorname{Tnfr}^{-/-}$mice lost weight and succumbed to HSV-1 infection at the same rate as

254 Gt and $\Delta$ CTT mice, whereas $T n f r 1^{-/-}$mice were as resistant to HSV-1 as WT mice (Fig.

255 5a and b). By contrast, $\operatorname{Irf}^{-/-}$mice presented an intermediate phenotype similar to that of

256 S365A mice. Viral loads in brain stems and total brain correlated with the disease

257 severity (Fig. 5c and Supp. Fig. S6a) and Ifnb expression in the brain stems was increased

258 only in WT mice (Fig. 5d). Ifnb expression was also reproducibly decreased in $T n f r 1^{-/}$

259 mice in vivo (but not in vitro Supp. Fig. S6b) for reasons that are currently unclear.

260 Nevertheless, these results suggest that S365A is critical for STING-induced IRF3

261 activation and Ifnb expression, but neither S365 nor IRF3 are essential for restriction of

262 HSV-1 replication in vivo, whereas the STING CTT and TBK1 are essential. 
a

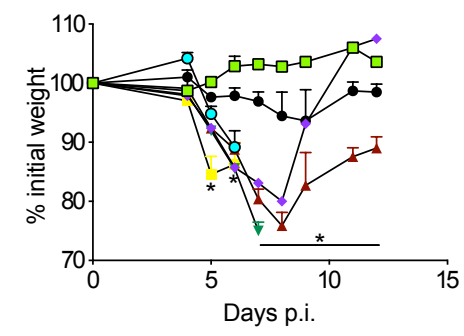

c

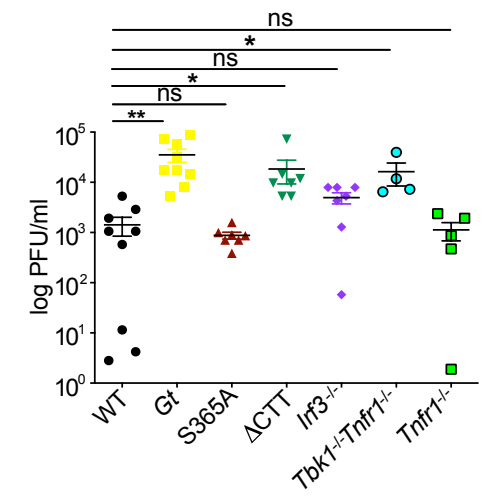

b

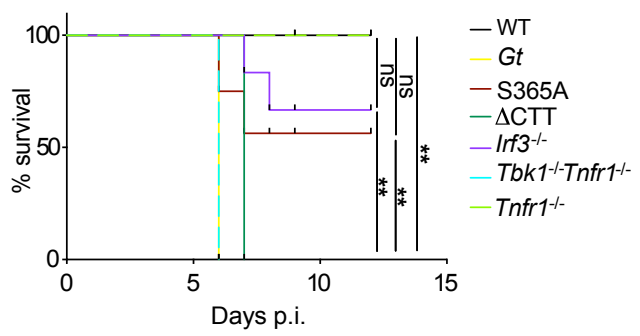

d

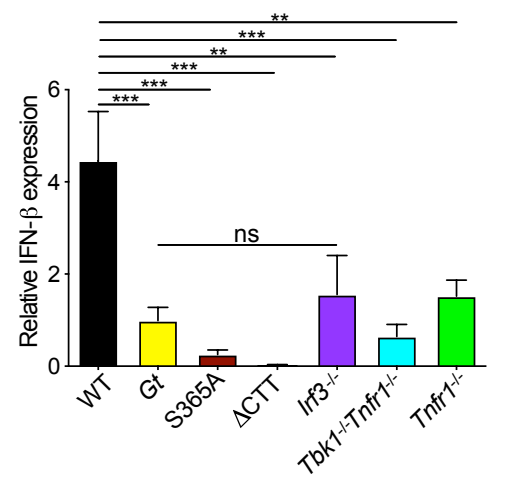

Figure 5. STING S365A and $\mathrm{Irf3}^{-/-}$mice phenocopy resistance to $\mathrm{HSV}-1$. Mice were ocular infected with $1 \times 10^{5}$ PFU of HSV-1 (strain 17). a, Percentage of initial weight following infection. b, Survival of infected mice. c, Viral titers in the brain stem. d, Relative Ifnb expression from brain stems. Representative results of at least three independent experiments. Error bars are SEM. Analyzed with one-way ANOVA and Tukey post-test. ${ }^{*}, \mathrm{p} \leq 0.05 ;{ }^{* *}, \mathrm{p} \leq 0.005 ; * * *, \mathrm{p} \leq 0.0001$. ns, not significant.

\section{Discussion}

The cGAS-STING pathway is a critical innate immune sensing pathway for the detection and elimination of DNA viruses, including HSV-1. STING activation leads to a variety of downstream antiviral responses, including IRF3-dependent induction of type I IFNs, as well as NF-kB activation and induction of autophagy responses. However, the relative contributions of these various STING-induced responses to host defense in vivo remains unclear. By generation and analysis of STING S365A and $\triangle \mathrm{CTT}$ mice, we were able to investigate the role of distinct STING-dependent signaling events during HSV-1 infection. Using both a systemic and an eye infection HSV-1 model, we found that 
STING $\triangle$ CTT mice. STING S365A mice failed to induce type I IFNs in response to HSV-1. We therefore propose that an interferon-independent function of STING is critical during HSV-1 infection in vivo. IRF3 is activated downstream of STING via recruitment to phospho-S365, and IRF3 is required for type I IFN induction by STING. Interestingly, we also found that $\operatorname{Irf} 3^{-/}$mice are relatively resistant to HSV-1 infection. By contrast, Gt, STING $\Delta$ CTT and $T b k 1^{-/-} \operatorname{Tnfr} 1^{-/-}$mice are fully susceptible to HSV-1. TBK1 recruitment and activation by STING requires the CTT but is independent of S365. Thus, we propose that the interferon- and IRF3-independent function of STING that protects against HSV-1 is initiated upon TBK1 recruitment by the STING CTT.

Although the exact mechanism that mediates protection to HSV-1 downstream of the STING CTT and TBK1 remains to be elucidated, we propose that a strong candidate is autophagy or an autophagy-like process. Indeed, we found that STING S365A is still able to induce the autophagy-like formation of LC3 puncta (Fig. 1d-f), a process previously shown also to require TBK1 $[28,52]$. Autophagy has previously been shown to be critical for control of HSV-1 [45]. However, it remains possible that an unidentified CTT-TBK1-induced response (other than, or in addition to, autophagy) is critical for STING-dependent control of HSV-1. Future studies are required to better elucidate the mechanism of STING-induced autophagy or other STING-induced responses, as there is no way at present to selectively eliminate STING-induced autophagy (or the putative autophagy-independent CTT-TBK1-dependent process). Nevertheless, our results clearly demonstrate the existence of effective S365/IRF3/interferon-independent antiviral functions for STING. 
Type I IFNs are essential for control of HSV-1 [41, 43, 46-48], a result we have confirmed (Supp. Fig. S6c-d). Thus, our results suggest only that STING-induced IFN, as opposed to all sources of type I IFN, is dispensable for resistance to HSV-1. Although we observe that most type I IFN induction during HSV-1 requires STING (Fig. 4f-i), other pathways for type I IFN induction (particularly the TLR3 pathway) [54-56] have been reported and appear to provide a low but essential type I IFN response.

Autophagy has been implicated in direct antiviral defense in many neurotropic viruses infections both in vivo and in vitro [45, 57-59]. In fact, HSV-1 has evolved different mechanisms to evade autophagy [58, 60, 61], but how STING activation initiates autophagy and whether STING-induced autophagy contributes to control of HSV-1 is not clear. In addition, the involvement of TBK1 during autophagy has been a matter of discussion. Some studies show that cells lacking TBK1 can still maintain autophagy-like events (LC3 conversion, puncta formation and autophagosomes formation) $[16,62]$ while other evidence suggests a critical role for TBK1 in phosphorylation of selective autophagy receptors and STING autophagosomal degradation $[63,64]$. Importantly, our data in primary cells suggest that TBK1 is needed for STING-mediated autophagy

One interesting feature of our results is that the STING S365A-independent protection we observe is delayed, especially in the eye infection model, and is coincident with the onset of adaptive $\mathrm{T}$ cell responses. Autophagy has been linked to induction of $\mathrm{T}$ cell responses [65-67]. Thus, one attractive possibility is that autophagy is required for antigen processing and presentation to elicit protective adaptive immune responses. Our 
newly generated STING mutant mice represent valuable tools to dissect this and other

putative IFN-independent functions of STING in vivo.

\section{References}

330 1. Janeway, C.A., Jr., Approaching the asymptote? Evolution and revolution in

2. Sorensen, L.N., et al., TLR2 and TLR9 synergistically control herpes simplex virus infection in the brain. J Immunol, 2008. 181(12): p. 8604-12.

3. Watson, R.O., et al., The Cytosolic Sensor cGAS Detects Mycobacterium tuberculosis DNA to Induce Type I Interferons and Activate Autophagy. Cell Host Microbe, 2015. 17(6): p. 811-819.

4. Watson, R.O., P.S. Manzanillo, and J.S. Cox, Extracellular M. tuberculosis DNA targets bacteria for autophagy by activating the host DNA-sensing pathway. Cell, 2012. 150(4): p. 803-15.

5. Gao, P., et al., Cyclic $\left[G\left(2^{\prime}, 5^{\prime}\right) p A\left(3^{\prime}, 5^{\prime}\right) p\right]$ is the metazoan second messenger produced by DNA-activated cyclic GMP-AMP synthase. Cell, 2013. 153(5): p. 1094-107.

6. Kranzusch, P.J., et al., Structure of human cGAS reveals a conserved family of second-messenger enzymes in innate immunity. Cell Rep, 2013. 3(5): p. 1362-8.

7. Sun, L., et al., Cyclic GMP-AMP synthase is a cytosolic DNA sensor that activates the type I interferon pathway. Science, 2013. 339(6121): p. 786-91.

8. Diner, E.J., et al., The innate immune DNA sensor cGAS produces a noncanonical cyclic dinucleotide that activates human STING. Cell Rep, 2013. 3(5): p. 1355-61.

9. Zhang, X., et al., Cyclic GMP-AMP containing mixed phosphodiester linkages is an endogenous high-affinity ligand for STING. Mol Cell, 2013. 51(2): p. 226-35.

10. Ablasser, A., et al., cGAS produces a 2'-5'-linked cyclic dinucleotide second messenger that activates STING. Nature, 2013. 498(7454): p. 380-4.

11. Cohen, D., et al., Cyclic GMP-AMP signalling protects bacteria against viral infection. Nature, 2019.

12. Margolis, S.R., S.C. Wilson, and R.E. Vance, Evolutionary Origins of cGASSTING Signaling. Trends Immunol, 2017. 38(10): p. 733-743.

13. Liu, Y., et al., Inflammation-Induced, STING-Dependent Autophagy Restricts Zika Virus Infection in the Drosophila Brain. Cell Host Microbe, 2018. 24(1): p. 57-68 e3.

14. Goto, A., et al., The Kinase IKKbeta Regulates a STING- and NF-kappaBDependent Antiviral Response Pathway in Drosophila. Immunity, 2018. 49(2): p. 225-234 e4.

15. Martin, M., et al., Analysis of Drosophila STING Reveals an Evolutionarily Conserved Antimicrobial Function. Cell Rep, 2018. 23(12): p. 3537-3550 e6.

16. Gui, X., et al., Autophagy induction via STING trafficking is a primordial function of the cGAS pathway. Nature, 2019. 567(7747): p. 262-266.

17. Sauer, J.D., et al., The N-ethyl-N-nitrosourea-induced Goldenticket mouse mutant reveals an essential function of Sting in the in vivo interferon response to Listeria monocytogenes and cyclic dinucleotides. Infect Immun, 2011. 79(2): p. 688-94. 
18. Liu, S., et al., Phosphorylation of innate immune adaptor proteins MAVS, STING, and TRIF induces IRF3 activation. Science, 2015. 347(6227): p. aaa2630.

19. Konno, H., K. Konno, and G.N. Barber, Cyclic dinucleotides trigger ULK1 (ATG1) phosphorylation of STING to prevent sustained innate immune signaling. Cell, 2013. 155(3): p. 688-98.

20. Tanaka, Y. and Z.J. Chen, STING specifies IRF3 phosphorylation by TBK1 in the cytosolic DNA signaling pathway. Sci Signal, 2012. 5(214): p. ra20.

21. Zhao, B., et al., A conserved PLPLRT/SD motif of STING mediates the recruitment and activation of TBK1. Nature, 2019. 569(7758): p. 718-722.

22. Zhang, C., et al., Structural basis of STING binding with and phosphorylation by TBK1. Nature, 2019. 567(7748): p. 394-398.

23. Motwani, M., S. Pesiridis, and K.A. Fitzgerald, DNA sensing by the cGAS-STING pathway in health and disease. Nat Rev Genet, 2019. 20(11): p. 657-674.

24. Tan, X., et al., Detection of Microbial Infections Through Innate Immune Sensing of Nucleic Acids. Annu Rev Microbiol, 2018. 72: p. 447-478.

25. Conlon, J., et al., Mouse, but not human STING, binds and signals in response to the vascular disrupting agent 5,6-dimethylxanthenone-4-acetic acid. J Immunol, 2013. 190(10): p. 5216-25.

26. Guo, F., et al., STING agonists induce an innate antiviral immune response against hepatitis B virus. Antimicrob Agents Chemother, 2015. 59(2): p. 1273-81.

27. Louis, C., C. Burns, and I. Wicks, TANK-Binding Kinase 1-Dependent Responses in Health and Autoimmunity. Front Immunol, 2018. 9: p. 434.

28. Saitoh, T., et al., Atg9a controls dsDNA-driven dynamic translocation of STING and the innate immune response. Proc Natl Acad Sci U S A, 2009. 106(49): p. 20842-6.

29. Rasmussen, S.B., et al., Activation of autophagy by alpha-herpesviruses in myeloid cells is mediated by cytoplasmic viral DNA through a mechanism dependent on stimulator of IFN genes. J Immunol, 2011. 187(10): p. 5268-76.

30. Manzanillo, P.S., et al., Mycobacterium tuberculosis activates the DNA-dependent cytosolic surveillance pathway within macrophages. Cell Host Microbe, 2012. 11(5): p. 469-80.

31. Collins, A.C., et al., Cyclic GMP-AMP Synthase Is an Innate Immune DNA Sensor for Mycobacterium tuberculosis. Cell Host Microbe, 2015. 17(6): p. 8208.

32. Wassermann, R., et al., Mycobacterium tuberculosis Differentially Activates cGAS- and Inflammasome-Dependent Intracellular Immune Responses through ESX-1. Cell Host Microbe, 2015. 17(6): p. 799-810.

33. Antonelli, L.R., et al., Intranasal Poly-IC treatment exacerbates tuberculosis in mice through the pulmonary recruitment of a pathogen-permissive monocyte/macrophage population. J Clin Invest, 2010. 120(5): p. 1674-82.

34. Dorhoi, A., et al., Type I IFN signaling triggers immunopathology in tuberculosissusceptible mice by modulating lung phagocyte dynamics. Eur J Immunol, 2014. 44(8): p. 2380-93.

35. Manca, C., et al., Virulence of a Mycobacterium tuberculosis clinical isolate in mice is determined by failure to induce Th1 type immunity and is associated with induction of IFN-alpha /beta. Proc Natl Acad Sci U S A, 2001. 98(10): p. 5752-7. 
36. Ji, D.X., et al., Type I interferon-driven susceptibility to Mycobacterium tuberculosis is mediated by IL-1Ra. Nat Microbiol, 2019.

37. Siqueira, M.D.S., R.M. Ribeiro, and L.H. Travassos, Autophagy and Its Interaction With Intracellular Bacterial Pathogens. Front Immunol, 2018. 9: p. 935.

38. Marinho, F.V., et al., The cGAS/STING Pathway Is Important for Dendritic Cell Activation but Is Not Essential to Induce Protective Immunity against Mycobacterium tuberculosis Infection. J Innate Immun, 2018. 10(3): p. 239-252.

39. Nakajima, A., et al., Cell type-dependent proapoptotic role of Bcl $2 L 12$ revealed by a mutation concomitant with the disruption of the juxtaposed Irf3 gene. Proc Natl Acad Sci U S A, 2009. 106(30): p. 12448-52.

40. Ishikawa, H., Z. Ma, and G.N. Barber, STING regulates intracellular DNAmediated, type I interferon-dependent innate immunity. Nature, 2009. 461(7265): p. 788-92.

41. Christensen, M.H., et al., HSV-1 ICP27 targets the TBK1-activated STING signalsome to inhibit virus-induced type I IFN expression. EMBO J, 2016. 35(13): p. 1385-99.

42. Reinert, L.S., et al., Sensing of HSV-1 by the cGAS-STING pathway in microglia orchestrates antiviral defence in the CNS. Nat Commun, 2016. 7: p. 13348.

43. Su, C., G. Zhan, and C. Zheng, Evasion of host antiviral innate immunity by HSV1, an update. Virol J, 2016. 13: p. 38.

44. Shen, G., et al., Herpes simplex virus 1 counteracts viperin via its virion host shutoff protein UL41. J Virol, 2014. 88(20): p. 12163-6.

45. Yordy, B., et al., A neuron-specific role for autophagy in antiviral defense against herpes simplex virus. Cell Host Microbe, 2012. 12(3): p. 334-45.

46. Leib, D.A., et al., Interferons regulate the phenotype of wild-type and mutant herpes simplex viruses in vivo. J Exp Med, 1999. 189(4): p. 663-72.

47. Wilcox, D.R., et al., The Type I Interferon Response Determines Differences in Choroid Plexus Susceptibility between Newborns and Adults in Herpes Simplex Virus Encephalitis. MBio, 2016. 7(2): p. e00437-16.

48. Ceron, S., et al., The STING agonist 5,6-dimethylxanthenone-4-acetic acid (DMXAA) stimulates an antiviral state and protects mice against herpes simplex virus-induced neurological disease. Virology, 2019. 529: p. 23-28.

49. Nicoll, M.P., J.T. Proenca, and S. Efstathiou, The molecular basis of herpes simplex virus latency. FEMS Microbiol Rev, 2012. 36(3): p. 684-705.

50. Steiner, I. and F. Benninger, Update on herpes virus infections of the nervous system. Curr Neurol Neurosci Rep, 2013. 13(12): p. 414.

51. Parker, Z.M., A.A. Murphy, and D.A. Leib, Role of the DNA Sensor STING in Protection from Lethal Infection following Corneal and Intracerebral Challenge with Herpes Simplex Virus 1. J Virol, 2015. 89(21): p. 11080-91.

52. Pilli, M., et al., TBK-1 promotes autophagy-mediated antimicrobial defense by controlling autophagosome maturation. Immunity, 2012. 37(2): p. 223-34.

53. Weidberg, H. and Z. Elazar, TBK1 mediates crosstalk between the innate immune response and autophagy. Sci Signal, 2011. 4(187): p. pe39.

54. Zhang, S.Y., et al., TLR3 deficiency in patients with herpes simplex encephalitis. Science, 2007. 317(5844): p. 1522-7. 
55. Reinert, L.S., et al., TLR3 deficiency renders astrocytes permissive to herpes simplex virus infection and facilitates establishment of CNS infection in mice. $\mathbf{J}$ Clin Invest, 2012. 122(4): p. 1368-76.

56. Zimmer, B., et al., Human iPSC-derived trigeminal neurons lack constitutive TLR3-dependent immunity that protects cortical neurons from HSV-1 infection. Proc Natl Acad Sci U S A, 2018. 115(37): p. E8775-E8782.

57. Orvedahl, A., et al., Autophagy protects against Sindbis virus infection of the central nervous system. Cell Host Microbe, 2010. 7(2): p. 115-27.

58. Orvedahl, A., et al., HSV-1 ICP34.5 confers neurovirulence by targeting the Beclin 1 autophagy protein. Cell Host Microbe, 2007. 1(1): p. 23-35.

59. Chaumorcel, M., et al., Human cytomegalovirus controls a new autophagydependent cellular antiviral defense mechanism. Autophagy, 2008. 4(1): p. 46-53.

60. Gobeil, P.A. and D.A. Leib, Herpes simplex virus gamma34.5 interferes with autophagosome maturation and antigen presentation in dendritic cells. MBio, 2012. 3(5): p. e00267-12.

61. Alexander, D.E., et al., Analysis of the role of autophagy in replication of herpes simplex virus in cell culture. J Virol, 2007. 81(22): p. 12128-34.

62. Liu, D., et al., STING directly activates autophagy to tune the innate immune response. Cell Death Differ, 2018.

63. Richter, B., et al., Phosphorylation of OPTN by TBK1 enhances its binding to Ub chains and promotes selective autophagy of damaged mitochondria. Proc Natl Acad Sci U S A, 2016. 113(15): p. 4039-44.

64. Prabakaran, T., et al., Attenuation of cGAS-STING signaling is mediated by a p62/SQSTM1-dependent autophagy pathway activated by TBK1. EMBO J, 2018. 37(8).

65. English, L., et al., Autophagy enhances the presentation of endogenous viral antigens on MHC class I molecules during HSV-1 infection. Nat Immunol, 2009. 10(5): p. 480-7.

66. Budida, R., et al., Herpes simplex virus 1 interferes with autophagy of murine dendritic cells and impairs their ability to stimulate CD8(+) T lymphocytes. Eur J Immunol, 2017. 47(10): p. 1819-1834.

67. Dengjel, J., et al., Autophagy promotes MHC class II presentation of peptides from intracellular source proteins. Proc Natl Acad Sci U S A, 2005. 102(22): p. 7922-7.

68. Wang, H., et al., One-step generation of mice carrying mutations in multiple genes by CRISPR/Cas-mediated genome engineering. Cell, 2013. 153(4): p. 9108.

69. Ishii, K.J., et al., TANK-binding kinase-1 delineates innate and adaptive immune responses to DNA vaccines. Nature, 2008. 451(7179): p. 725-9.

70. Mayer-Barber, K.D., et al., Caspase-1 independent IL-1beta production is critical for host resistance to mycobacterium tuberculosis and does not require TLR signaling in vivo. J Immunol, 2010. 184(7): p. 3326-30.

71. Posel, C., et al., Isolation and Flow Cytometric Analysis of Immune Cells from the Ischemic Mouse Brain. J Vis Exp, 2016(108): p. 53658. 
Acknowledgements: We thank members of the Vance, Barton, Stanley and Cox labs for discussions, L. Flores, P. Dietzen and R. Chavez for technical assistance, H. Nolla and A. Valeros and the Cancer Research Laboratory for flow cytometry. We thank Dr. Mary West and Dr. Pingping He of the High-Throughput Screening Facility (HTSF) at UC Berkeley. This work was performed in part in the HTSF, which provided the Perkin Elmer Opera Phenix, funded by NIH Instrument Grant S10OD021828 with the assistance of Christopher Noel. We thank Chris Bowen and Daniel Renner in the Szpara lab for assistance with viral genome sequencing and analysis. R.E.V. was supported by Investigator in the Pathogenesis of Infectious Diseases awards from the Burroughs Wellcome Fund. R.E.V. is an HHMI Investigator and is supported by NIH grants AI075039 and AI066302. The authors declare no competing interests.

\section{Materials and Methods}

Viruses and reagents. Dulbecco's Modified Eagle Medium (DMEM) was obtained from Gibco and supplemented with $100 \mathrm{U} / \mathrm{ml}$ penicillin, $100 \mathrm{mM}$ streptomycin and LPS-free FCS (BioWhittaker). DAPI, TRIzol, Poly I:C (all from Invitrogen) Lipofectamine 2000 (Invitrogen) were used in the experiments described below. HSV-1 (strains KOS and strain 17) was grown in Vero cells. The Vero cells used were from the lab stock. The titers of the stocks used were $8-14 \times 10^{9} \mathrm{PFU} / \mathrm{ml}$. Titers were determined by TCID50 assay on Vero cells. Both strains were used for infection of mice, while only KOS strain was used for in vitro stimulation.

Mice. All mice used were specific pathogen free, maintained under a $12 \mathrm{~h}$ light-dark cycle ( 7 am to $7 \mathrm{pm}$ ), and given a standard chow diet (Harlan irradiated laboratory animal diet) ad libitum. Wild type C57BL/6J mice were originally obtained from the Jackson Laboratories (JAX). CRISPR/Cas9 targeting was performed by both pronucleus and cytoplasm injection of Cas 9 mRNA, sgRNA, and repair template oligos into fertilized zygotes from C57BL/6J female mice (JAX, stock no. 000664), essentially as described previously[68]. STING S365A mice were generated by targeting exon 8 from 
STING introducing an AGT (serine) to GCC (alanine) substitution at codon 365 . The sgRNA sequence was 5' - GCTGATCCATACCACTGATG - 3' and the repair template oligo was

\section{C*A*G*ACAAGGCTGTCCCATGCCTCAGATGAGGTCAGTGCGGAGTGGGAGA} GGCTGATCCATACCGGCGATGAGGAGTCTTGGCTCTTGGGACAGTACGGAGG GAGGAGGTGCCACTGA*G*G*T (underlined is the PAM location). For STING $\triangle \mathrm{CTT}$ mice, valine 340 was replaced by a premature stop codon. The sgRNA sequence was 3' - GGAGGAAAAGAAGGACTGCT - 5' and the repair template oligo was C*C*C*ACAGACGGAAACAGTTTCTCACTGTCTCAGGAGGTGCTCCGGCACAT TCGTCAGGAAGAAAAGGAGGAGTGAACCATGAATGCCCCCATGACCTCAGTG GCACCTCCTCCCTCC ${ }^{*} \mathrm{G}^{*} \mathrm{~T}^{*} \mathrm{~A}$ (underlined is the PAM location). The asterisks indicate phosphorothioate linkages in the first and last three nucleotides. $\operatorname{Irf} 3^{-/-}$mice were generated by targeting exon 6 from IRF3. The sgRNA sequence was 5' - GAGGTGACCGCCTTCTACCG - 3'. Founder mice were genotyped as described below, and founders carrying mutations were bred one generation to $\mathrm{C} 57 \mathrm{BL} / 6 \mathrm{~J}$ mice to separate modified haplotypes. Homozygous lines were generated by interbreeding

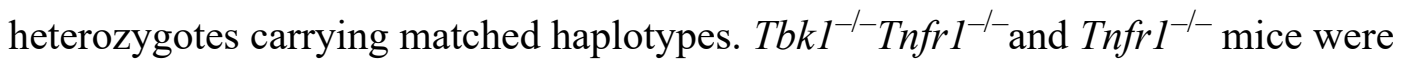
described elsewhere[69].

Preparation of gRNA transcript. DNA oligos (IDT, Coralville, NY) were heated to $95^{\circ} \mathrm{C}$ followed by cooling down to room temperature. The self-annealing oligo duplex was cloned into linearized T7 gRNA vector (System Biosciences, Mountain View, CA USA). The cloned sgRNA was sequence verified by DNA sequencing. Then sgRNA template for in vitro transcription (IVT) was prepared by PCR amplification of Phusion high fidelity DNA polymerase (NEB Biolabs, Ipwich, MA), the PCR mixture was cleaned up by PCR cleanup reaction (Qiagen, Hilden, Germany). The sgRNA transcripts were generated by IVT synthesis kit (System Biosciences, Palo Alto, CA). Quality of sgRNA transcripts was analyzed by NanoDrop (Thermo Fisher Scientific, Waltham, MA) and Bioanalyzer instrument (Agilent Technologies, Inc., Santa Clara, CA).

Genotyping of STING S365A, $\Delta$ CTT and Irf3 alleles. Exon 8 of STING and Exon 6 of Irf3 were amplified by PCR using the following primers (all 5' to 3'): S365A fwd: CCA ACC ATT GAA GGA AGG CTC AGT C, S365A rev: CTC ACT GTC TCA GGA GGT 

GCT CC; $\triangle$ CTT fwd: CTA GAG CCC AGA CAA GGC TGT CC, $\triangle$ CTT rev: CCC ACA GAC GGA AAC AGT TTC TCA C; Irf3 fwd: AAC GTG AGT GCC AGC TGT GG, Irf3 rev: CTT CAC AAG CTT GTC CGT CAG AAA CC. Primers were used at 200nM in a reaction with $2.5 \mathrm{mM} \mathrm{MgCl} 2$ and $75 \mu \mathrm{M}$ dNTPs and 1 Unit Taq polymerase (Thermo Fisher Scientific) per reaction. Cleaned PCR products were diluted 1:16 and sequenced using Sanger sequencing (Berkeley DNA Sequencing facility).

Cell culture. Macrophages were derived from the bone marrow of C57BL/6J or STING mutant (Gt, S365A or $\Delta \mathrm{CTT})$ mice. Macrophages were derived by 7 days of culture in RPMI 1640 medium supplemented with 10\% serum, $100 \mathrm{mM}$ streptomycin, $100 \mathrm{U} / \mathrm{ml}$ penicillin, $2 \mathrm{mM}$ L-glutamine and 10\% supernatant from 3T3-M-CSF cells, with feeding on day 5. Mouse primary microglia cells and astrocytes were isolated and cultured from the cerebrum of P0 pups. Neonatal cerebra were trypsinized for $20 \mathrm{~min}$ and filtered through a $70 \mu \mathrm{m}$ pore size filter. Cells of 3 cerebrum were seeded on one poly-d-lysinecoated $75 \mathrm{~cm}^{2}$ culture flask and incubated with DMEM containing 10\% FCS. The medium was replaced on day 2 after plating. Henceforth, either microglia or astrocytes were isolated. Astrocytes were isolated using the following method: after 7 days of culture, cells were shaken for 30 minutes, supernatant was aspirated, and the remaining adherent cells were predominantly astrocytes. Purity of each population was determined by FACS. Primary dissociated hippocampal cultures were prepared from postnatal day 01 (P0-1). Mice were euthanized using standard protocols. Briefly, bilateral hippocampi from 2-3 pups were dissected on ice and pooled together. The tissue was dissociated using $34.4 \mathrm{ug} / \mathrm{ml}$ papain in dissociation media (HBSS Ca2+, $\mathrm{Mg} 2+$ free, $1 \mathrm{mM}$ sodium pyruvate, $0.1 \%$ D-glucose, 10mM HEPES buffer) and incubated for $3 \mathrm{~min}$ at $37^{\circ} \mathrm{C}$. The papain was neutralized by incubation in trypsin inhibitor $(1 \mathrm{mg} / \mathrm{ml}$ in dissociation media) at $37^{\circ} \mathrm{C}$ for $4 \mathrm{~min}$. After incubation, the dissociation media was carefully removed and the tissue was gently triturated, manually, in plating media (MEM, 10\% FBS, $0.45 \%$ DGlucose, $1 \mathrm{mM}$ sodium pyruvate, $1 \mathrm{mM}$ L-glutamine). Cell density was counted using a TC10 Automated cell counter (Biorad). For western blot experiments, 2.2-2.5 $\times 10^{5}$ cells were plated onto 24- well plates pre-coated with Poly-D-Lysine (PDL) (Corning) in $500 \mathrm{ul}$ of plating media. After 3 hours, plating media was removed and 800ul maintenance media (Neurobasal media (GIBCO) with 2mM glutamine, pen/strep, and B-27 
supplement (GIBCO)) was added per well. After 4 days in vitro 1uM cytosine arabinoside (Sigma) was added to prevent glial proliferation. Neurons were maintained in maintenance media for 14 days with partial media changes every 4 days. For immunofluorescence, $2 \times 10^{3}$ cells were plated in pre-coated 96 well plates (CellCarrier96 Ultra Microplates, black, PerkinElmer) following the same procedure.

Murine M. tuberculosis infections. M. tuberculosis strain Erdman (gift of S.A. Stanley) was used for all infections. Frozen stocks of this wild-type strain were made from a single culture and used for all experiments. Cultures for infection were grown in Middlebrook 7H9 liquid medium supplemented with 10\% albumin-dextrose-saline, $0.4 \%$ glycerol and $0.05 \%$ Tween- 80 for five days at $37^{\circ} \mathrm{C}$. Mice were aerosol infected using an inhalation exposure system (Glas-Col, Terre Haute, IN). A total of $9 \mathrm{ml}$ of culture was loaded into the nebulizer calibrated to deliver $\sim 400$ bacteria per mouse as measured by colony forming units (CFUs) in the lungs 1 day following infection (data not shown). Mice were sacrificed at various days post-infection as indicated in the figure legends to measure CFUs and/or cytokines. All lung lobes were homogenized in PBS plus 0.05\% Tween- 80 or processed for cytokines (see below), and serial dilutions were plated on 7H11 plates supplemented with 10\% oleic acid, albumin, dextrose, catalase (OADC) and $0.5 \%$ glycerol. CFUs were counted 21-25 days after plating.

Cytokine measurements. Cell-free lung homogenates from M. tuberculosis infected mice were generated as previously described[70]. Briefly, lungs were dissociated through $100 \mu \mathrm{m}$ Falcon cell strainers in sterile PBS with 1\% FBS and Pierce Protease Inhibitor EDTA-free (Thermo Fisher). An aliquot was removed for measuring CFU by plating as described above. Cells and debris were then removed by first a low-speed centrifugation (approximately $300 \times \mathrm{g}$ ) then a high- speed centrifugation (approximately $2000 \times \mathrm{g}$ ) and the resulting cell-free homogenate was filtered twice with $0.2 \mu \mathrm{m}$ filters to remove all $M$. tuberculosis for work outside of BSL3. All homogenates were aliquoted, flash-frozen in liquid nitrogen and stored at $-80^{\circ} \mathrm{C}$. Each aliquot was thawed a maximum of twice to avoid potential artifacts due to repeated freeze-thaw cycles. All cytokines were measured using Cytometric Bead Assay (BD Biosciences) according to manufacturer protocols. TNF- $\alpha$ from DMXAA and LPS stimulated mice was also measured by CBA. Results 
were collected using BD LSRFortessa (BD Biosciences) and analyzed using GraphPad Prism v6.0c. TNF- $\alpha$ from primary macrophages supernatant was measured by ELISA.

\section{Murine HSV-1 infection models}

Intravenous infection. Age and sex matched (7-10-week old) mice were warmed under a lamp for venous dilation and inoculated with $1 \times 10^{6}$ PFU HSV-1 (KOS strain) in $200 \mu 1$ of PBS or mock infected with PBS only.

Ocular infection. Age and sex matched (7-10-week old) mice, were anaesthetized with intraperitoneal (i.p.) injection of ketamine $(100 \mathrm{mg} / \mathrm{kg}$ body weight) and xylazine $(10 \mathrm{mg} / \mathrm{kg}$ body weight). Corneas were scarified using a $25 \mathrm{G}$ needle and mice were either inoculated with $1 \times 10^{5}$ PFU HSV-1 (strain 17) in $5 \mu \mathrm{l}$, or mock infected with $5 \mu \mathrm{l}$ of PBS. Eyewash was collected by gently proptosing each eye and wiping a sterile cotton swab around the eye in a circular motion. The swabs were placed in $0.5 \mathrm{ml}$ of DMEM medium and stored at $-80{ }^{\circ} \mathrm{C}$ until the titer was determined. Whole brains, brain stems, spinal cords and livers were frozen immediately at $-80^{\circ} \mathrm{C}$. Tissues were homogenized with tissue homogenizer (Polytron PT $2500 \mathrm{E}$ ) for $2 \mathrm{~min}$ at frequency 10. Tissues were used for RNA isolation with TRIzol or used for virus titration.

Scoring and tissue harvest. Mice were scored for disease, weighed at the indicated times post infection and euthanized at the specified times post infection for tissue harvesting or once they met end point criteria. The scoring was performed as blinded study, largely following previous descriptions by others[51] with the following minor modifications: symptoms related to neurological disease named body condition score (BCS) (0: normal, healthy 1: hunched, 2: uncoordinated, lethargic, mild paralysis, 3: unresponsive/no movement, complete paralysis).

Infection and cell stimulations (transfections). For infections, bone marrow derived macrophages from $\mathrm{C} 57 \mathrm{BL} / 6 \mathrm{~J}$ mice were plated at $1-2 \times 10^{6}$ cells/well. The next day they were stimulated with cyclic dinucleotides c-di-GMP, 2'3'cGAMP, Sendai virus (SeV) and poly I:C. Cells were transfected using Lipofectamine 2000 (LF2000; Invitrogen) according to the manufacturer's protocol. All cyclic dinucleotides nucleic acid stimulants were mixed with LF2000 at a ratio of $1 \mu \mathrm{LF} 2000 / 1 \mu \mathrm{g}$ nucleic acid, incubated at room temperature for 20-30 min, and added to cells at a final concentration of $4 \mu \mathrm{g} / \mathrm{ml}$ (6-well plates). For Sendai Virus, cells were infected at 150 hemagglutination units (HAU)/ml. 
For poly I:C, $2 \mathrm{mg} / \mathrm{ml}$ of the stock solution was heated at $50^{\circ} \mathrm{C}$ for $10 \mathrm{~min}$ and cooled to room temperature before mixing with LF2000. Transfection experiments were done for 6 $\mathrm{h}$, unless otherwise stated in the figures.

Immunoblotting. BMMs were seeded at a density of $1 \times 10^{6}$ cells per well in 6 well tissue culture plates and transfected the next day using Lipofectamine 2000 (Invitrogen) according to the manufacturer's instruction. Cells were lysed at indicated time post transfection with radioimmunoprecipitation assay (RIPA) buffer supplemented with 2 $\mathrm{mM}$ NaVO3, $50 \mathrm{mM}$ b-Glycerophosphate, $50 \mathrm{mM} \mathrm{NaF}, 2 \mathrm{mM}$ PMSF, and Complete Mini EDTA-free Protease Inhibitor (Roche). Proteins separated with denaturing PAGE and transferred to Immobilon-FL PVDF membranes (Millipore). Membranes were blocked with Li-Cor Odyssey blocking buffer. Primary antibodies were added and incubated overnight. Primary antibodies used were: anti-TBK1 (D1B4) (\#3504), antiphospho-TBK1/NAK (Ser172) (D52C2) (\#5483), anti-STING (D2P2F) (\#13647), antiphospho-STING (Ser366) (D7C3S) (\#19781), anti-phospho-IRF3 (Ser396) (4D4G) (\#4947), all purchased from Cell Signaling Technologies. Anti-IRF3 (EP2419Y) (\#ab76409) was from Abcam. Secondary anti-rabbit IgG was conjugated to Alexa Fluor680 (Invitrogen). Immunoblots were imaged using a Li-Cor fluorimeter.

Quantitative PCR. Stimulated cells were overlayed with TRIzol (Invitrogen) and stored. RNA was isolated according to the manufacturer's protocol and was treated with RQ1 RNase-free DNase (Promega). $0.5 \mu \mathrm{g}$ RNA was reverse transcribed with Superscript III (Invitrogen). SYBRGreen dye (ThermoFisher Scientific) was used for quantitative PCR assays and analyzed with a real-time PCR system (StepOnePlus; Applied Biosystems). All gene expression values were normalized to Rps17 (mouse) levels for each sample. The following primer sequences were used: mouse Ifnb, (forward) 5'ATAAGCAGCTCCAGCTCCAA-3' and (reverse) 5'-CTGTCTGCTGGTGGAGTTCA3'; mouse Rps 17, (forward) 5'-CGCCATTATCCCCAGCAAG-3' and (reverse) 5'TGTCGGGATCCACCTCAATG-3'; mouse Viperin, (forward) 5'TTGGGCAAGCTTGTGAGATTC-3' and (reverse) 5'TGAACCATCTCTCCTGGATAAGG-3'; mouse TNF, (forward) 5'TCTTCTCATTCCTGCTTG TGG-3' and (reverse) 5'-GGTCTGGGCCATAGAACTGA- 
3'; mouse $I L-6$, (forward) 5'-GCTACCAAACTGGATATAATCAGGA-3' and (reverse) 5'-CCAGGTAGCTATGGTACTCCAGAA-3'.

Immunofluorescence and high-content imaging. Bone marrow derived macrophages were transfected with 0.2 ug of Cy3-labeled DNA for 6 hours. Cells were washed with PBS, fixed in 4\% paraformaldehyde and ice-cold methanol. Cells were washed 3x with PBS and blocked and permeabilized with 2\% BSA and 0.3\% Triton X100. LC3 puncta staining was performed using mouse monoclonal antibody (Nanotools, catalog \#0260100/LC3-2G6 at 1:400, RT) for 3hours, followed by secondary goat anti-mouse IgG labeled with Alexa Fluor 488 (Life Technologies at 1:4000, RT) for 1 hour. Nuclei were stained with DAPI. For imaging, cells in 96-well plates were imaged using an Opera Phenix (Perkin Elmer) at RT, using a $\times 401.1$ NA water immersion lens (Zeiss). Images were exported to Harmony High-Content Imaging and Analysis Software and automated colocalization measurements were performed with the Perkin Elmer Harmony software package. A pipeline was created to measure colocalization of Cy3-labeled DNA and LC3. Quantification was performed using data collected from 16 fields per well in 96-well format. Data was then analyzed in Prism using one-way ANOVA analysis.

Flow cytometry. Single suspensions were prepared from each experimental group using a modified protocol as described[71]. To analyze tetramer positive cells, cell suspensions were stained with the following cell surface antibodies: CD3e (clone 145-2C11, BD Horizon), CD8a (clone 53-6.7, Biolegend), CD45 (clone 30-F11, eBioscience), CD44 (clone IM7, eBioscience), CD11b (clone M1/70, eBioscience), MHCII I-A/I-E (cloneM5/114.15.2, Biolegend), CD19 (clone eBio1D3, eBioscience), CD45R (B220) (clone RA3-6B2, Invitrogen), Ly6G (Gr-1) (clone 1A8-Ly6g, eBioscience). Samples were acquired on a FACS X20 Fortessa (BD Bioscience) and analyzed with FlowJo software (TreeStar).

Statistical analysis. All data were analyzed with one-way ANOVA test and Tukey posttest unless otherwise noted and survival data were analyzed with Log-rank (Mantel-Cox) test. Both tests were run using GraphPad Prism 6. *, p $\leq 0.05 ;{ }^{* *}, \mathrm{p} \leq 0.005,{ }^{* * *}, \mathrm{p} \leq$ 0.0001 . All errors bars are SEM and all center bars indicate means. 
bioRxiv preprint doi: https://doi.org/10.1101/2019.12.12.874792; this version posted December 13, 2019. The copyright holder for this preprint (which was not certified by peer review) is the author/funder. All rights reserved. No reuse allowed without permission.

719

720

721

722

723

724

725

726

727

728

729

730

731

732

733

734

735

736

737

738

739

740

741

742

743

744

745

746

747

748

749 


\section{$750 \quad$ Supplementary figures}

a

... LeuLeuIleSerGlyMetAspGlnPro... translation of coding strand

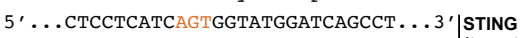
3'... . GAGGAGTAGTCACCATACCTAGTCGGA... .5' (target locus)

3'....gagGagtagcGGCCATACCTAGTCGg...5'

b

...GluLysGlugluValThrMetAsnAla... translation of coding strand 5 '....GAAAAGGAGGAGGTTACCATGAATGCC...3 3' STING

$3^{\prime}$... TTTTTCCTCCTCCAATGGTACTTACGG...5' (target locus)

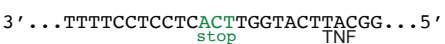

C

e
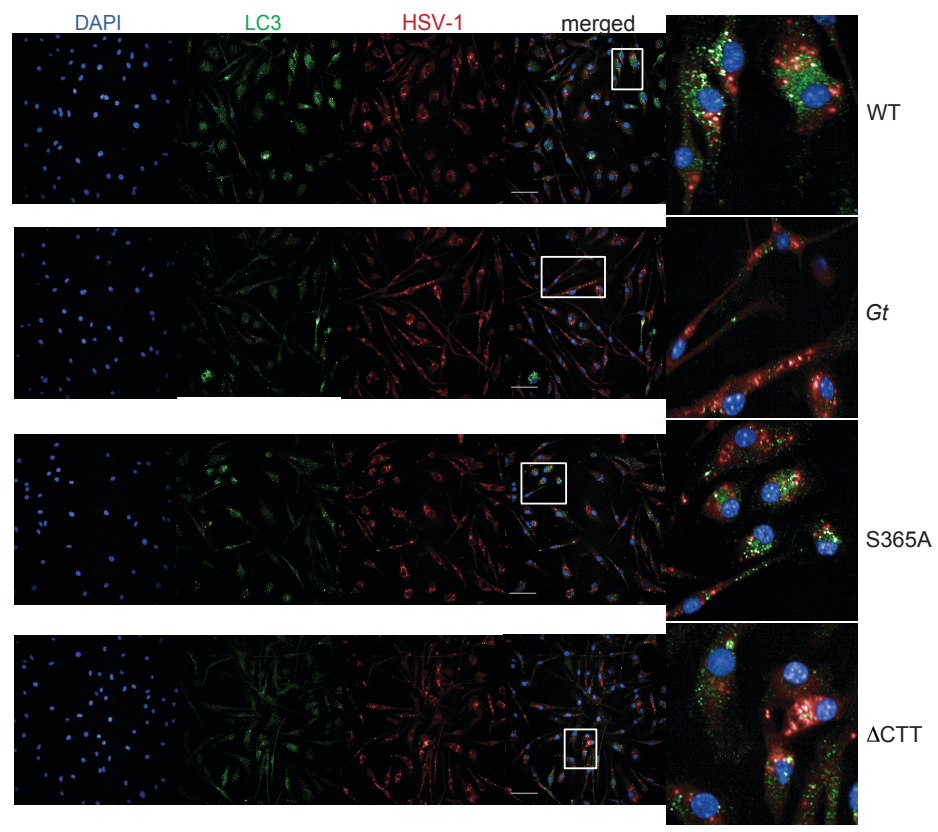

WT

Gt

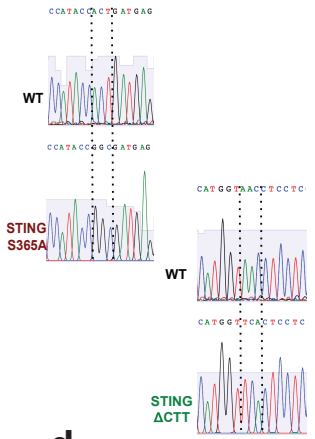

d

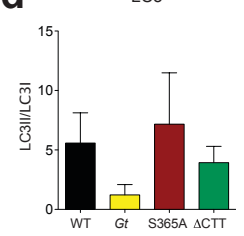

Fig. S1. (related to figure 1). a, Creation of STING S365A and b, $\Delta$ CTT mice using CRISPR/Cas9. c, Bone marrow derived macrophages were stimulated for $6 \mathrm{~h}$ and TNF- $\alpha$ was measured on the supernatant. d, Quantification of Fig.1d using LC3II/LC3I ratio. e, Colocalization of DNA and LC3 is increased in WT and S365A cells. Fluorescence images of primary macrophages transfected for $6 \mathrm{~h}$ with Cy3-labeled DNA and LC3. Images were analyzed by an automated pipeline created on Perkin Elmer Harmony software for colocalization quantification (for more details refer to Methods). Scale bars are $50 \mu \mathrm{m}$. 


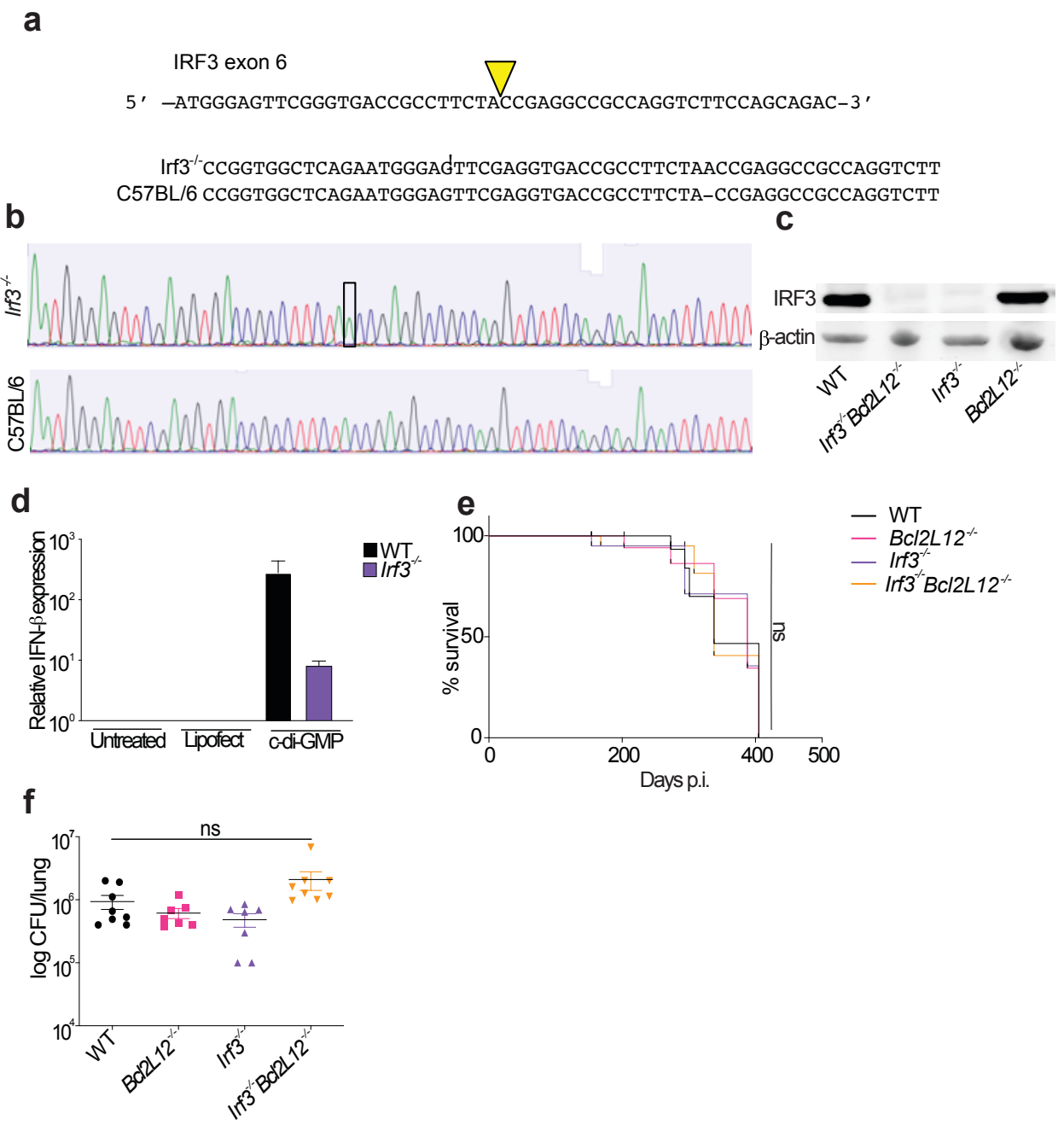

Fig. S2. (related to figure 2). Creation of IRF3 deficient mice using CRISPR/Cas9. a, CRISPR/Cas9 targeting strategy for IRF3. b, Sequencing of the targeted locus resulting in $\mathrm{Irf3}^{-/}$mutation. c, Immunoblot of MEFs for IRF3. d, Primary macrophages were transfected with c-di-GMP for $6 \mathrm{~h}$ and relative expression of Ifnb was analyzed. e, Mice were aerosol infected with $400 \mathrm{CFU}$ dose of M. tuberculosis (Erdman strain). Survival of infected mice. f, Bacterial burden from lungs at 21 days post infection. All mice except C57BL/6J WT were bred in-house. Representative results of four independent experiments. Error bars are SEM. Analyzed with one-way ANOVA and Tukey post-test.

770 ns, not significant. 
b

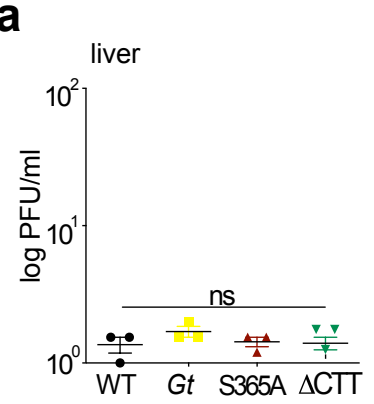

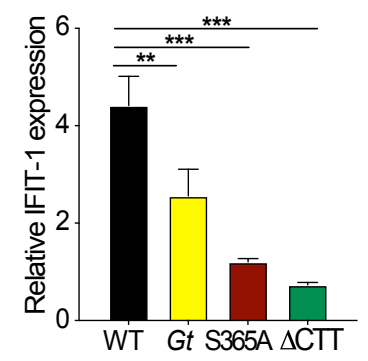

C

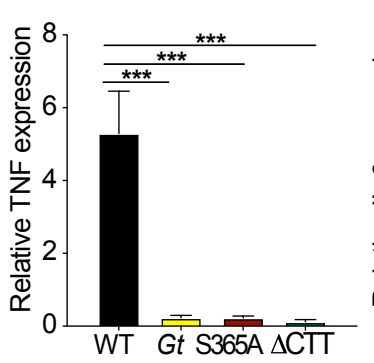

d

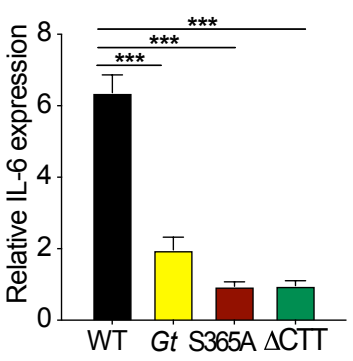

Fig. S3. (related to figure 3). Mice were intravenously infected with $1 \times 10^{6} \mathrm{PFU}$ of HSV1 (KOS strain). a, Viral titers in the liver at 6 days p.i. b, Relative expression of IFIT-1 $\mathbf{c}$, TNF and d, IL-6 from brains at 3 days p.i. All mice except C57BL/6J WT were bred inhouse. Representative results of five independent experiments. Error bars are SEM. Analyzed with one-way ANOVA and Tukey post-test. **, $\mathrm{p} \leq 0.005$; $^{* * *}, \mathrm{p} \leq 0.0001$. ns, not significant.

a eye wash

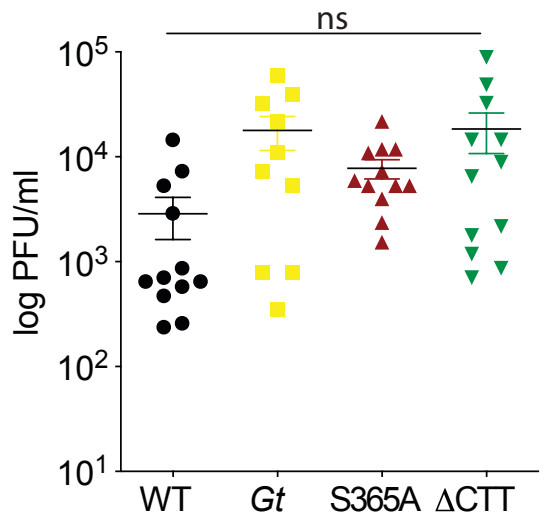

b Viperin brain stem

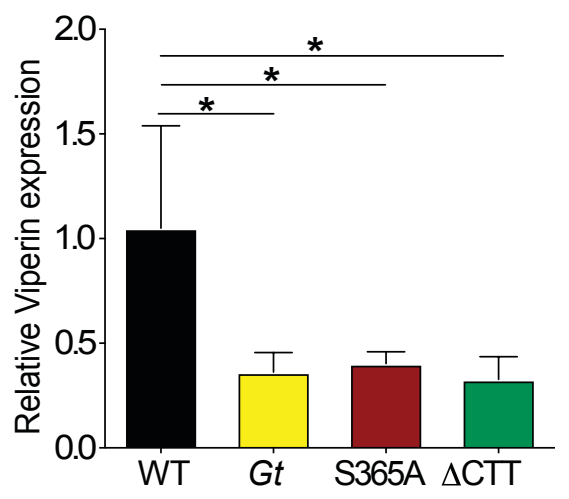

Fig. S4. (related to figure 4). Mice were ocular infected with 1x10 PFU of HSV-1 (strain 17). a,Viral titers from eyes washed at 2 days p.i.. b, Relative expression of viperin. All mice except C57BL/6J WT were bred in-house. Representative results of three independent experiments. Error bars are SEM. Analyzed with one-way ANOVA and Tukey post-test. ns, not significant. 
a

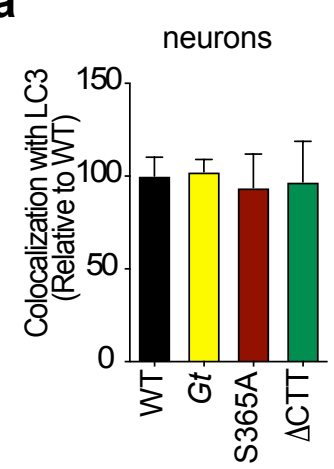

b

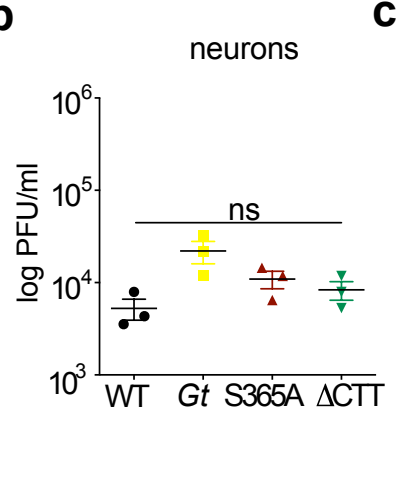

C

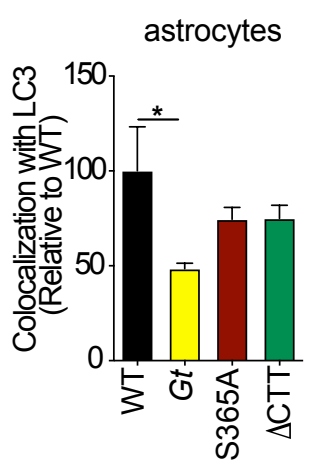

d

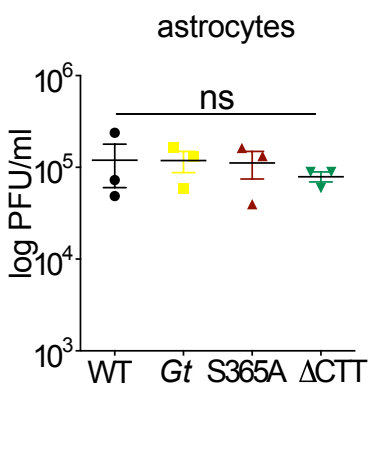

e

astrocytes

neurons

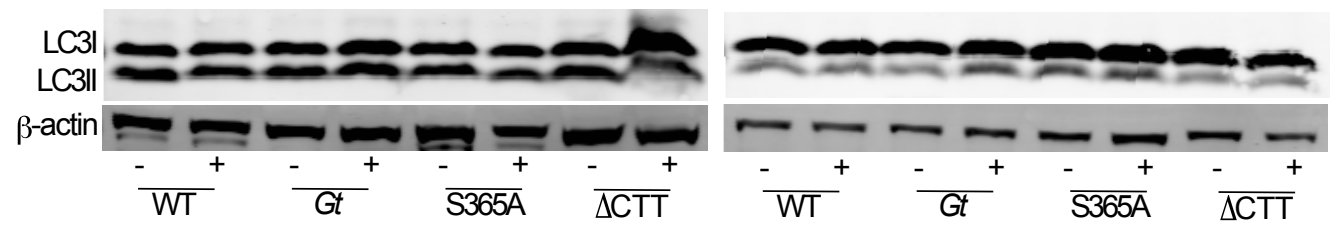

Fig. S5. (related to figure 4). Brain cells (neurons and astrocytes) were harvested from P0 pups and infected with HSV-1 (KOS strain) at a MOI 1 for 6 h and later were stained for LC3 and HSV-1. a, Quantification of colocalization of LC3-HSV-1 in neurons was performed and $\mathbf{b}$, Viral titers from supernatants were collected $48 \mathrm{~h}$ later and quantified by TCID50 assay. c-d, Same as a-b, in astrocytes. e, Cell lysates were collected at $4 \mathrm{~h}$ postinfection and immunoblot for LC3 and $\beta$-actin was performed. Representative results from two independent experiments. Error bars are SEM. Analyzed with one-way ANOVA and Tukey post-test. $*, p \leq 0.05$. ns, not significant. 

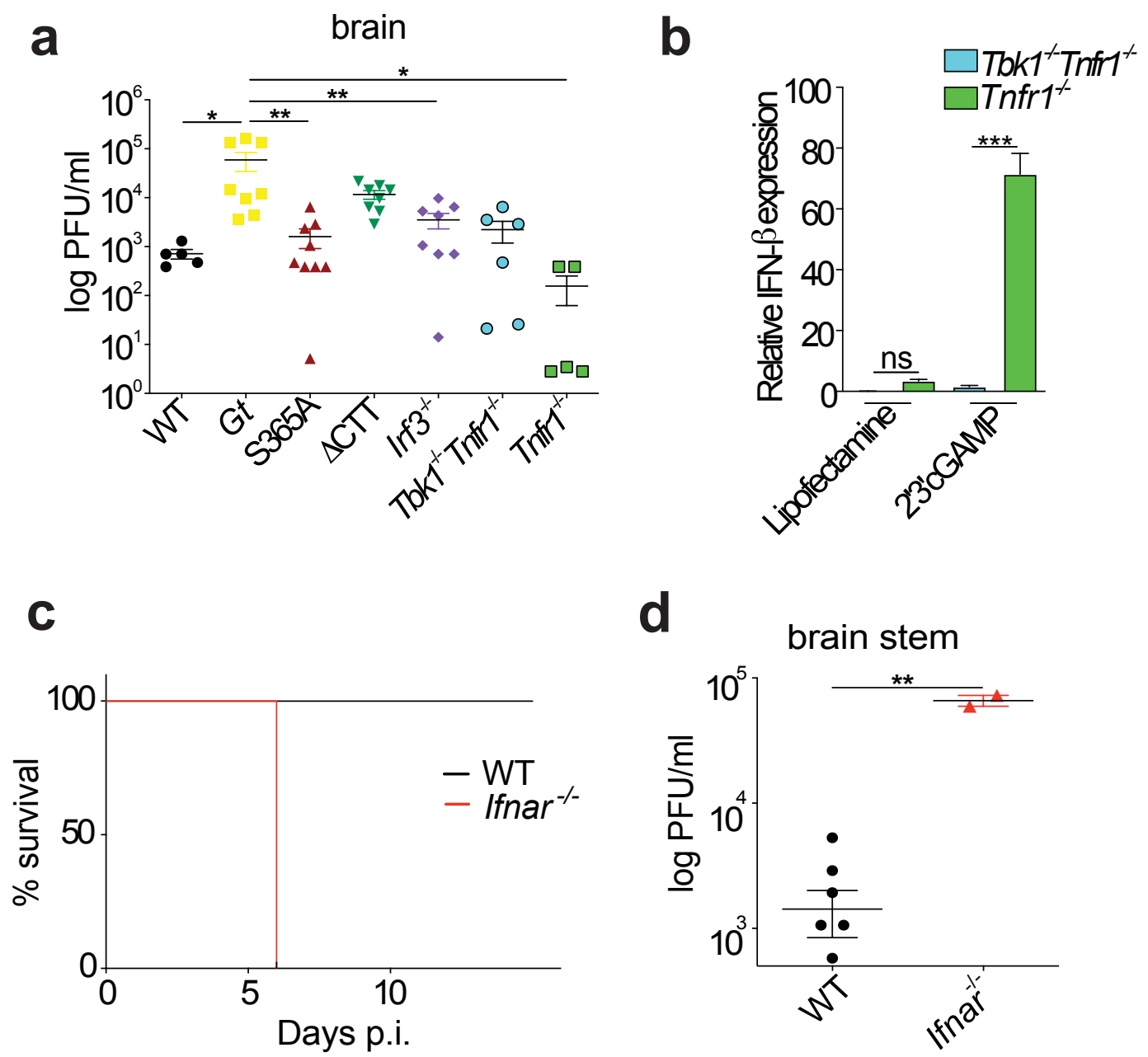

Fig. S6. (related to figure 5). a, Mice were ocular infected with $1 \times 10^{5}$ PFU of HSV-1 (strain 17) and viral titers measured in the brain 6 days p.i. b, BMDMs were transfected with 2'3'cGAMP for $6 \mathrm{~h}$ and relative expression of Ifnb was analyzed. c, Mice were ocular infected with $1 \times 10^{5} \mathrm{PFU}$ of HSV-1 (strain 17) and survival rate and $\mathbf{d}$, viral titers measured in the brain stem 6 days p.i. All mice except C57BL/6J WT were bred inhouse. Representative results of two independent experiments. Error bars are SEM. Analyzed with one-way ANOVA and Tukey post-test. *, $\mathrm{p} \leq 0.05 ; * *, \mathrm{p} \leq 0.005$. ns, not significant. 